\title{
Point clouds by SLAM-based mobile mapping systems: accuracy and geometric content validation in multisensor survey and stand-alone acquisition
}

\author{
Giulia Sammartano $^{1}$ (D) $\cdot$ Antonia Spanò ${ }^{1}$
}

Received: 15 January 2018 / Accepted: 3 May 2018 /Published online: 1 June 2018

(C) The Author(s) 2018

\begin{abstract}
The paper provides some operative replies to evaluate the effectiveness and the critical issues of the simultaneous localisation and mapping (SLAM)-based mobile mapping system (MMS) called ZEB by GeoSLAM ${ }^{\mathrm{TM}}$ https://geoslam.com/technology/. In these last years, this type of handheld 3D mapping technology has increasingly developed the framework of portable solutions for closerange mapping systems that have mainly been devoted to mapping the indoor building spaces of enclosed or underground environments, such as forestry applications and tunnels or mines. The research introduces a set of test datasets related to the documentation of landscape contexts or the 3D modelling of architectural complexes. These datasets are used to validate the accuracy and informative content richness about ZEB point clouds in stand-alone solutions and in cases of combined applications of this technology with multisensor survey approaches. In detail, the proposed validation method follows the fulfilment of the endorsed approach by use of root mean square error (RMSE) evaluation and deviation analysis assessment of point clouds between SLAM-based data and 3D point cloud surfaces computed by more precise measurement methods to evaluate the accuracy of the proposed approach. Furthermore, this study specifies the suitable scale for possible handlings about these peculiar point clouds and uses the profile extraction method in addition to feature analyses such as corner and plane deviation analysis of architectural elements. Finally, because of the experiences reported in the literature and performed in this work, a possible reversal is suggested. If in the 2000s, most studies focused on intelligently reducing the light detection and ranging (LiDAR) point clouds where they presented redundant and not useful information, contrariwise, in this sense, the use of MMS methods is proposed to be firstly considered and then to increase the information only wherever needed with more accurate high-scale methods.
\end{abstract}

Keywords 3D mapping $\cdot$ Cultural heritage $\cdot$ Sensor integration $\cdot$ SLAM $\cdot$ ZEB $\cdot$ Mobile mapping systems $\cdot$ Landscape

\section{Introduction}

The concepts corresponding to the pursuit of rapid mapping solutions for the cultural heritage $(\mathrm{CH})$ domain in supporting multiscale documentation of indoor and/or outdoor built heritage should be more tailored on several needs that may arise in individual operative contexts, particularly, historic structures and

Giulia Sammartano

giulia.sammartano@polito.it

Antonia Spanò

antonia.spano@polito.it

1 Dipartimento di Architettura e Design (DAD), Politecnico di Torino, Viale Mattioli 39, 10129 Turin, Italy landscape scenarios. Similarly, the methodological approaches and technological solutions offered by geomatics research should comply with time-cost ratios in the overall resource consumption within a context that is as prone to the underinvestment of funds as the $\mathrm{CH}$ one. These solutions are essential whenever monitoring documentation operations are frequently required in cases of reduced accessibility to spaces or wherever the possibility of implementing the consolidated 3D survey procedures would be limited or not enough for wider areas.

Thus, the effectiveness of imaging or ranging measurement systems for 3D data acquisition can be evaluated in their adaptability to complex settings and based on the achievable descriptive capabilities in richly geometrically featured environments or enclosed spaces for a suitable surface reconstruction responsive to the expected purposes. In this regard, the proposed research tests a new rapid mapping technology: a 3D 
handheld mobile system based on the simultaneous localisation and mapping (SLAM) algorithm applied to various profile measurements, in which the new system is called the ZEB scanner by GeoSLAM (marketed in Italy by Me.s.a srl). In fact, beyond the close-range photogrammetry (CRP) imaging approach, new worthy solutions have overlooked the panorama of ranging systems to produce dense and detailed point clouds.

The currently pervasive image-matching photogrammetric methods provide even more effective densification algorithms and flexible and user-friendly solutions in 3D reconstruction both in terrestrial and in unmanned aerial vehicle (UAV) aerial datasets (Lingua et al. 2017; Murtiyoso et al. 2017; Remondino et al. 2014).

Also, the range-based techniques being currently implemented are many types of light detection and ranging (LiDAR)-based mobile mapping systems (MMSs) (Puente et al. 2013; Rodríguez-Gonzálvez et al. 2017), and they are flexible and competitive solutions for the 3D mapping of wide and complex spaces (Farella et al. 2016; Tucci et al. 2017) compared with the close-/medium-/long-range laser devices used for terrestrial laser scanning (TLS). Nevertheless, these MMSs remain a pricey and complex technological solution of fusion-based sensors with different levels of equipment and manageability. The research and development on these integrated systems have helped address movability convenience issues that better meet the needs of applicability in $\mathrm{CH}$ sites according to their potential needs related to their complexities.

In the recent research, the developments in the framework of portability and compactness features for MMS solutions in close-range mapping systems have been mainly devoted to transportable solutions. Handheld or by otherwise portable devices can be thus deployed to survey indoor volumes and enclosed or narrow environments, such as multilevel buildings, industrial spaces, forestry, tunnels, mines, caves, and all related environmental and geospatial applications. Several studies have also investigated these portable technologies in the $\mathrm{CH}$ domain.

Thus, the portable handheld MMS, the ZEB SLAM-based scanner, in the ZEB1 and ZEB-REVO configurations provided by GeoSLAM ${ }^{\mathrm{TM}}$, has been tested in distinctive configurations in the proposed datasets and has been studied in this work. The first dataset is from the San Silvestro archaeomining park in Livorno, Tuscany, Italy (Brocchini et al. 2017), with its ancient underground mining network and ruined masonry fortress, while the second dataset is from the Valperga castle in Torino, Piedmont, Italy (Chiabrando et al. 2017a, b), with the system of spaces articulated in above- and underground volumes. The historical complexes are featured by peculiar architectural geometries and material surfaces.

Indoor and outdoor scenes have been made available to validate point cloud accuracy in surface reconstruction, computed by the mapping system along different trajectories: first, the trajectories are evaluated based on their stand-alone use and on their abilities to support and integrate the geometric description provided by other methodological image-based or range-based approaches.

\section{Mobile mapping systems in $\mathrm{CH}$ domain complexity}

The LiDAR-based MMSs offer a very wide range of competitive solutions for the 3D mapping of extensive and/or complex spaces. They can be generally defined as combinations of the following sensors for point cloud acquisition and positioning (or geo-positioning): a mapping sensor (i.e., LiDAR, active 3D imaging systems), an inertial measurement unit, a global navigation satellite system (GNSS) receiver, and a time referencing unit (Puente et al. 2013).

According to the acquisition mode movement, platforms can be divided on those movements that equip a vehicle (by ground, on air, or on water) or portable movements (by towing trolley, man-portable backpacks, or portable handheld devices), with or without GNSS positioning solutions (Nocerino et al. 2017).

The LiDAR-based embedded ranging measurements integrated with the different navigation system solutions in the MMS equipping cars or mobile/self-propelled platforms allow the helpful exploitation of derived point clouds for their analysis and management by specific multidisciplinary activities regarding heritage structures.

Especially recently, the vehicle-equipping mobile navigation systems seem to be an effective answer to cover sizable distances and obtain metrically dense point cloud surfaces with geometric and radiometric data. Their use in dense urban centres of historical cities, featured by high structures and narrow streets, has been studied for Bergamo city in Toschi et al. (2017) and Toschi et al. (2015), to actually demonstrate the point cloud ability to reach the detail for highscale accuracy; the fruitful integration with aerial datasets can provide a dense $3 \mathrm{D}$ reconstruction on which the $3 \mathrm{D}$ city modelling approaches can obtain a fundamental metric source of geometric data. In larger outdoor complexes, the use of an MMS can also be a valid complementary integration or alternative for 3D mapping purposes and to document a very extensively built heritage complex in advantageous times, as proposed in the impressive fortified walls of Avila Alcázar in Rodríguez-Gonzálvez et al. (2017). Similarly, the integrability aspects of dense surfaces, retrieved by different MMSs, are being studied for their validation and point cloud optimisation for several uses in $\mathrm{CH}$ documentation and management. In fact, the ability of mobile ranging systems to cover great complexes at different scales within the integration of other different static and dynamic measurement techniques and related instrumental solutions can be a significant contribution 
in the multiscale documentation of richly architectonic featured sites. This aptitude is investigated in the multiscale combined workflow in Tucci et al. (2017), where, in parallel to imaging and ranging approaches (CRP, TLS, UAV photogrammetry), a multisource range-based 3D mapping method was implemented by fourfold MMS solutions, a mobile system equipping a car, a portable ranging measurement trolley, a portable backpack, and a handheld portable solution that allows accurate 3D mapping of an articulated system of underground passageways.

\section{Handheld MMS for SLAM-based 3D mapping}

Within the framework of portable scanners, this last type of handheld 3D mapping solution, quite unique of its kind, is the ZEB by GeoSLAM. The solution of the indoor positioning problem is based on implementing a SLAM-based algorithm applied on range-based profiles progressively extracted by the head of the continuously moving device in particularly enclosed and richly geometrically featured spaces (Bosse and Zlot 2009; Bosse et al. 2012), as explained in "ZEB system operational behaviour".

The main application fields in which the portable systems firstly proved their effectiveness have been those ones in which the use of their operating principle could be better exploited: forestry (Bauwens et al. 2016; Ryding et al. 2015); civil applications and geology, such as underground tunnels (Dewez et al. 2016; Eyre et al. 2016); and open pit mines (Vanneschi et al. 2017).

Recently, this handheld SLAM-based MMS has also been specifically evaluated and metrically tested with other similar technological solutions for indoor/outdoor structure mobile mapping, as proposed in Thomson et al. (2013), in Sirmacek et al. (2016) and Díaz-Vilariño et al. (2017), and in Nocerino et al. 2017.

Parallelly, the crucial ability of the ZEB system to recover also large outdoor manufactured spaces and articulated structures has been examined and is still being studied. The use of ZEB was first proposed in Zlot et al. (2014), towards the scattered historical structures distributed in the extensive natural environment in the Peel Island Lazaret despite the state of conservation of the ruined artefacts in such a scenario. In works by Sammartano (2017) and Sammartano (2018), the ZEB 3D mapping approach is proposed and validated rather with the use of UAV photogrammetric survey as a valuable combination for the damage documentation of collapsed structures by rapid mapping in risk areas and post-disaster scenarios. The details achievable by this ranging terrestrial approach overtake in most cases the photogrammetric approach, despite the lack in the reconstruction of the upper parts: the integration of the essential aerial approach is needed to be geometrically completed.

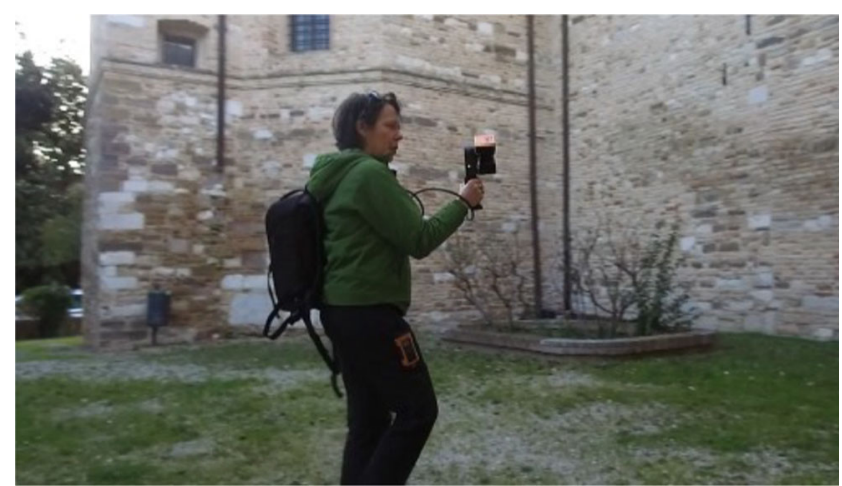

Fig. 1 The ZEB-REVO system with the rotating ranging head, tested by Geomatics Lab for Cultural Heritage, PoliTo, in 2017 in San Severino Marche (MC)

Many other contexts belonging to the built urban heritage, in the outdoor scenario but mostly in the indoor settings similar to underground environments such as tunnels and caves, have offered challenging validation purposes, such as the twofold technique proposed for this validation research in "Experimental section: validation strategy".

The use of the ZEB1 system inside the Pausilypon site, in Farella et al. (2016), returned a flexible solution for documenting the enclosed passageways with topographic variations along lengthy trajectories in viable operation time. However, this system emphasises the problem in the final accuracy validation based on both the risk related to the subdivision of an elongated path and the problem of regular and low geometrically featured surfaces that do not allow the SLAM-based algorithm to optimally operate for the progressive profile alignment. The use of the ZEB1 mapping device in similar indoor enclosed and mazy spaces is validated as a winning approach in terms of time-consuming performances, manoeuvrability, and continuity in surface reconstruction,

Table 1 Main specs of the ZEB devices by GeoSLAM, from https:// geoslam.com/technology/, Cadge 2016, Eyre et al. 2016, and Nocerino et al. 2017

\begin{tabular}{lll}
\hline & ZEB1 & ZEB-REVO \\
\hline Wavelength & $905 \mathrm{~nm}$ & $905 \mathrm{~nm}$ \\
Eye-safe laser & Class1 & Class1 \\
Laser speed & $40 \mathrm{~Hz}$ & $100 \mathrm{~Hz}$ \\
Laser lines & 40 lines $/ \mathrm{s}$ & 100 lines $/ \mathrm{s}$ \\
Scan speed & $\times 1$ & $\times 2.5$ \\
Maximum range & $15-30 \mathrm{~m}$ & $15-30 \mathrm{~m}$ \\
Points density & $\sim 43,200 \mathrm{pps}$ & $\sim 43,200 \mathrm{pps}$ \\
3D measurement & $\pm 0.1 \%$ & $\pm 0.1 \%$ \\
$\quad$ declared accuracy & & $270^{\circ} \mathrm{H}_{\mathrm{FOV}} / 100^{\circ} \mathrm{V}_{\mathrm{FOV}}$ \\
FoV & - & $\sim 2 \mathrm{~kg}$ \\
Weight/portability & $\sim 1.5 \mathrm{~kg}$ & \\
$\quad$ (head + data logger) & & \\
\hline
\end{tabular}


Fig. 2 The output data of ZEB acquisition: the time-marked $3 \mathrm{D}$ model, with related range colour scale (a) and the quality-marked trajectory along the castle courtyard (b). Shaded view of the 3D model (c)

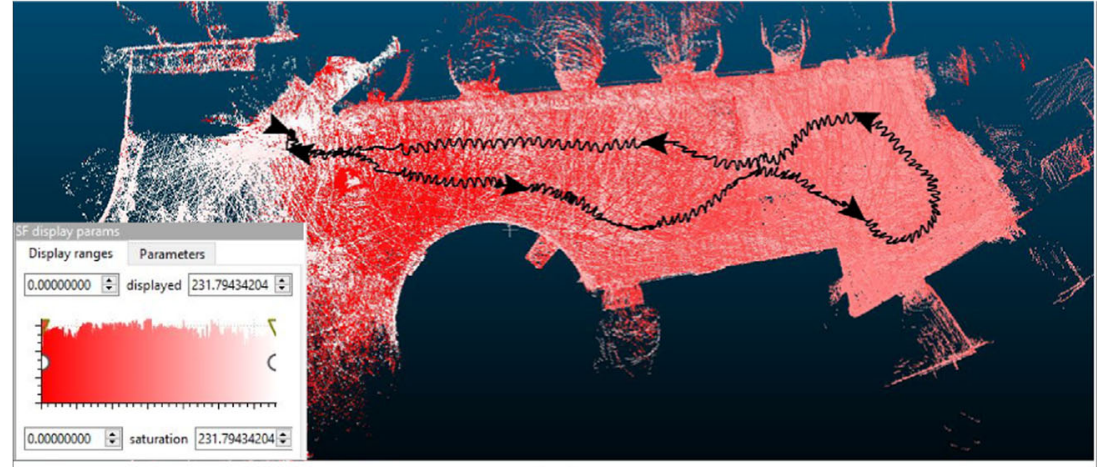

(a)

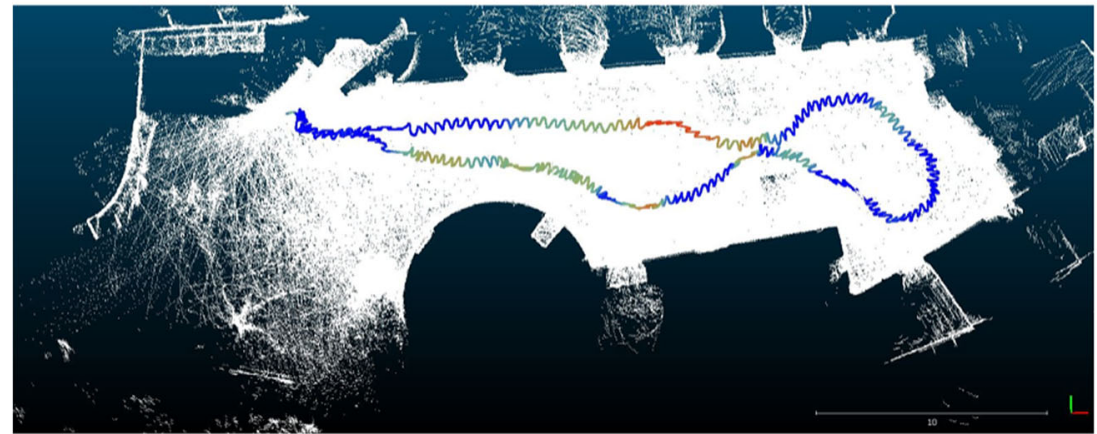

(b)

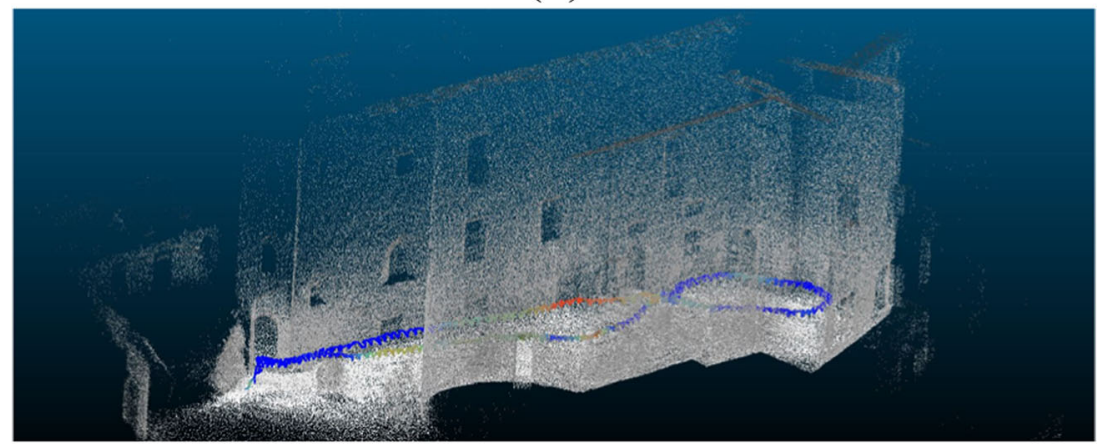

(c) such as in roof garrets resulting from the extrados of the vaults of the transept in Milan's cathedral, in Mandelli et al. (2017).

The proficiency of this technological solution to accomplish many kinds of complexity requisites in these kinds of $\mathrm{CH}$ framework can be briefly summarised in terms of complexity of applicative contexts and feasible applications:

- Physical complexity of contexts

- Articulated landscape complexes or particularly wide sites to be travelled through several paths configurations, such as variables regarding linear or circular trajectories with uphill/downhill and roundtrip configurations

- Structured built heritage in indoor and outdoor developments, underground environments, ramblings, and narrow surface reconstructions
- Application complexity

- Rapid performance, avoiding time-consuming operations

- Possibly replacing consolidated systems in indoor configurations

Table 2 Framework of the selected dataset subject to validation

\begin{tabular}{lll}
\hline & Indoor & Outdoor \\
\hline Valperga castle context & $\begin{array}{l}\text { Cylindrical tower (A) } \\
\text { Ancient ice house (B) }\end{array}$ & Courtyard (C) \\
$\begin{array}{c}\text { San Silvestro } \\
\text { archaeominig park }\end{array}$ & $\begin{array}{c}\text { Medieval mining } \\
\text { cave (D) } \\
\text { Stand-alone validation }\end{array}$ & Fortified village (E) \\
\hline
\end{tabular}


- Need to obtain essential volumetric features for planning the design of in-depth specific elements and/or insightfocused analyses in single parts

- Capability to deepen and specify the geometric content of digital surface models (DSMs) defined by other measurement approaches (i.e., UAV photogrammetry documentation), targeting the scale of surveying included among architectural and urban scales (between 1:100 and 1:200)

\section{ZEB system operational behaviour}

The ZEB system by GeoSLAM (Fig. 1) is essentially based on a moving head equipped with a ranging measurement laser capturing 2D point profiles, without a GNSS receiver or direct RGB data. The system also comprises an inertial measurement unit with triaxial gyros, accelerometers, and three-axis magnetometers.
In the ZEB1 device, the sensor head is mounted on a spring that freely and passively swings during operator and vehicle movement (Bosse et al. 2012). In the ZEBREVO-implemented solution, the head is regularly rotating automatically during the operator motion (Table 1). In the last update of ZEB-REVO, the aspects related to the acquisition and interactivity of the operator with the data collection have been enhanced as the in-time visibility of profile SLAM-based registration on a handheld device coupled with the scanning body during the acquisition. The RGB GoPro action cam is now equipped to the scanning body for the post-texturing of point clouds, as already proposed for ZEB1 by Nocerino et al. (2017) and Zlot et al. (2014).

The ranging sensor is a 2D lightweight pulse (Hokuyo UTM-30LX scanner (Nikoohemat et al. 2017)) continuously emitted in the form of pulsed light beams in the near infrared at 905-nm wavelength. The travelling time of flight of the pulse from the sensor to the object and back provides the range
Fig. 3 Valperga castle tower: a slam-based point cloud and coloured trajectory, b segmented CRP cloud, and $\mathbf{c}$ derived 2D section drawing

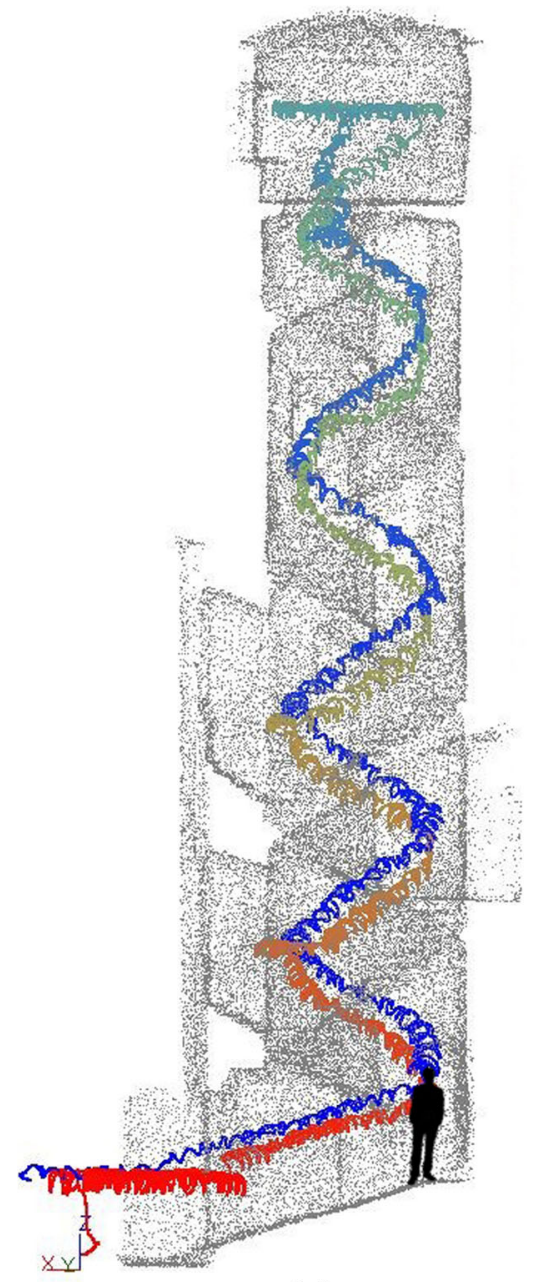

(a)

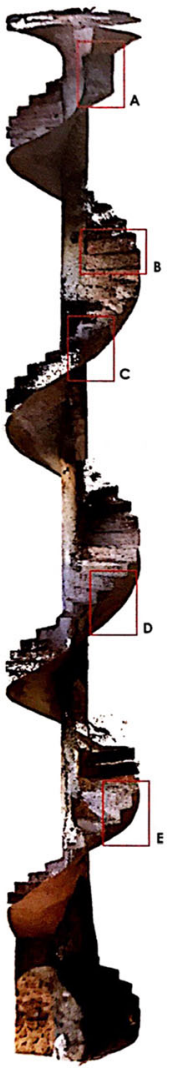

(b)

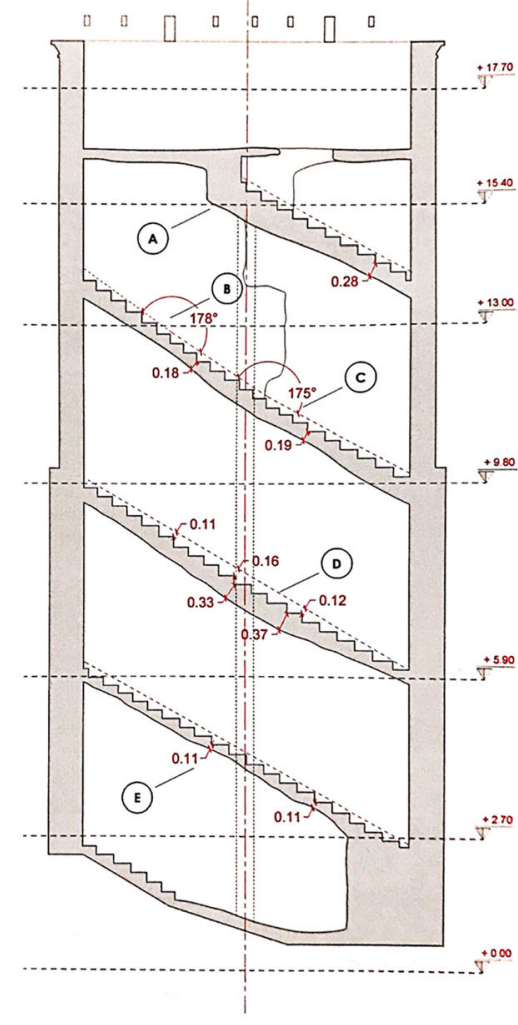

(c) 
measurement collecting profiles of about 43,200 pps at $40 \mathrm{~Hz}$ for ZEB1 and $100 \mathrm{~Hz}$ for ZEB-REVO (Cadge 2016; Dewez et al. 2016). They are simultaneously aligned during the trajectory by the registration algorithm implemented in the system and based on 3D SLAM robotics technology (Riisgaard 2005).

The specific SLAM algorithm first developed by the CSIRO ICT Centre in Brisbane (Australia) (Bosse et al. 2012; GeoSLAM 2016) is based on the exploitation of geometrical attributes featuring the surveyed environment, and the algorithm works for both the trajectory incremental motion estimation and global PC registration along the trajectory. The trajectory $[T(\tau)]$ is estimated by the algorithm by sequences of translation $t(\tau)$ and rotation $r(\tau)$ functions of time $(\tau)$ according to six DOF (degrees of freedom). The raw laser profiles continuously captured into time-windowed segments are progressively re-projected in the $3 \mathrm{D}$ reconstruction according to the best correspondence to surface characterisation, indicating that profile matching is an ICP (iterative closest point)-like approach (Bellekens et al. 2014). These iterative process conditions ensure during the ZEB acquisition the continuity of profile registration with the previous time segment, minimising errors between matching surfaces and deviations from the measured inertial measurement unit accelerations and rotational velocities and guaranteeing the precision of the global registration.

The resulting data processed either by GeoSLAM proprietary software (SW) application running on a local machine or by a pay-as-you-go process via cloud processing is a double kind of point cloud comprising the $3 \mathrm{D}$ reconstruction and the reference trajectory from which it is generated. Both these point-based data are potentially time-marked, as shown in Fig. 2a, and it is possible to show in range colours the trajectory of the estimated quality of the SLAM-based profile registration, as shown in Fig. $2 b$. Problems related to registration drift errors can be corrected by point cloud reprocess and merging in the last 2017 release of the GeoSLAM Hub SW tools.

The fundamental topics considered to avoid bad quality in registering point cloud profiles are mainly related to the operative fieldwork:

- Initialisation procedure is mandatory on a planar surface, and the return trip arrival point must be in the same position as the starting point. This is fundamental for the point cloud alignment and closure.
Fig. 4 Castle courtyard and overlooking buildings: a alignment of the ZEB points with LiDAR model (in violet, the underground ice cellar position); b vertical view of the starting portion of ice cellar point cloud with red trajectory (red box)

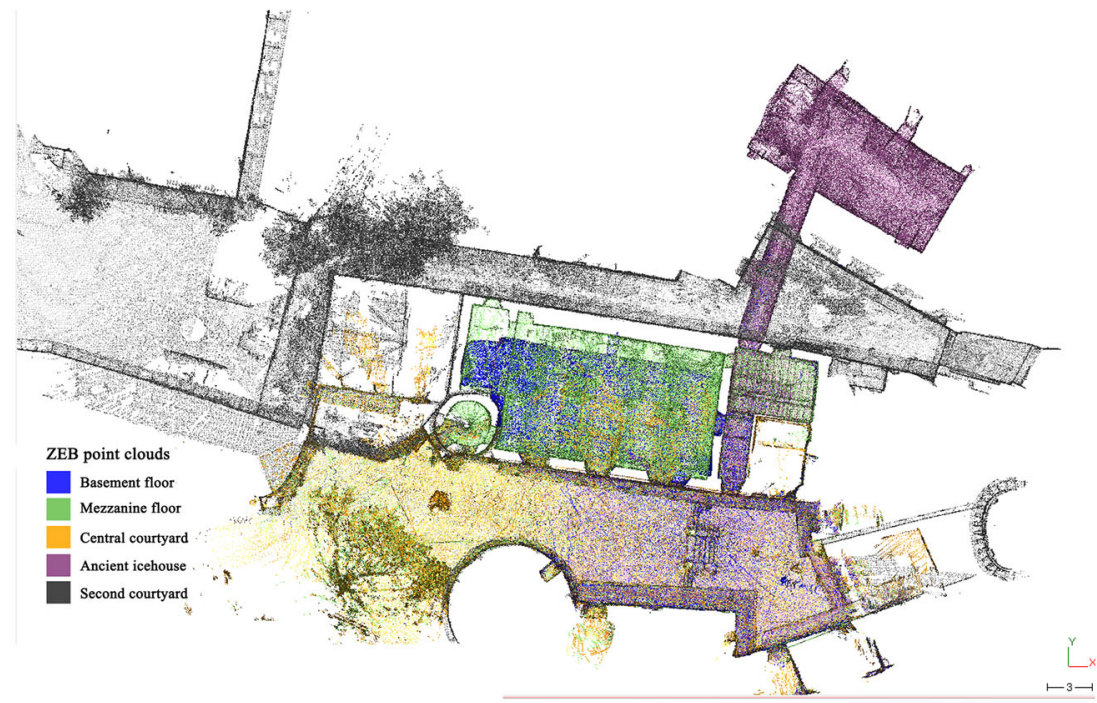

(a)

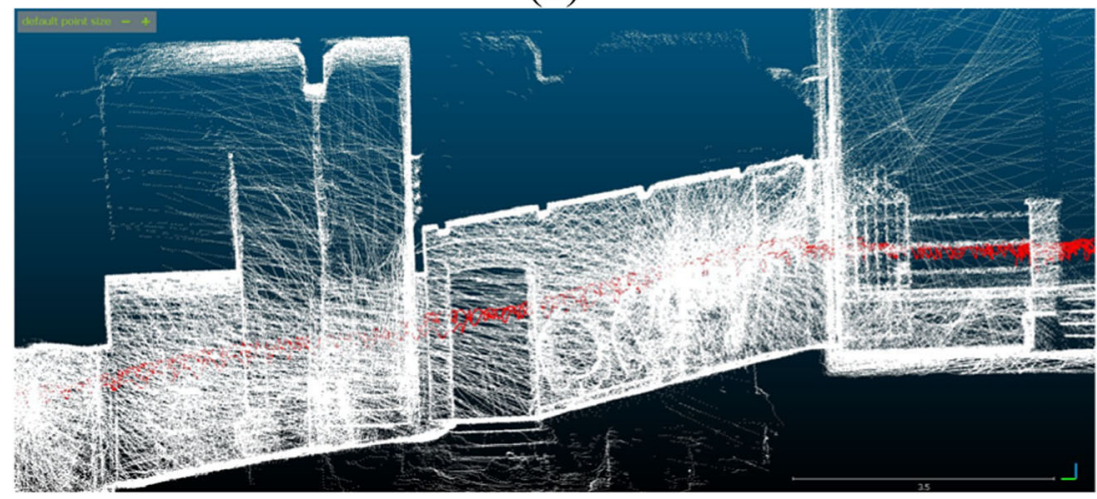

(b) 
Fig. 5 a Excerpt of the north side of the courtyard TLS 3D model with the integration of the roofing elements from UAV. b Dense 3D model derived from oblique and nadiral UAV images

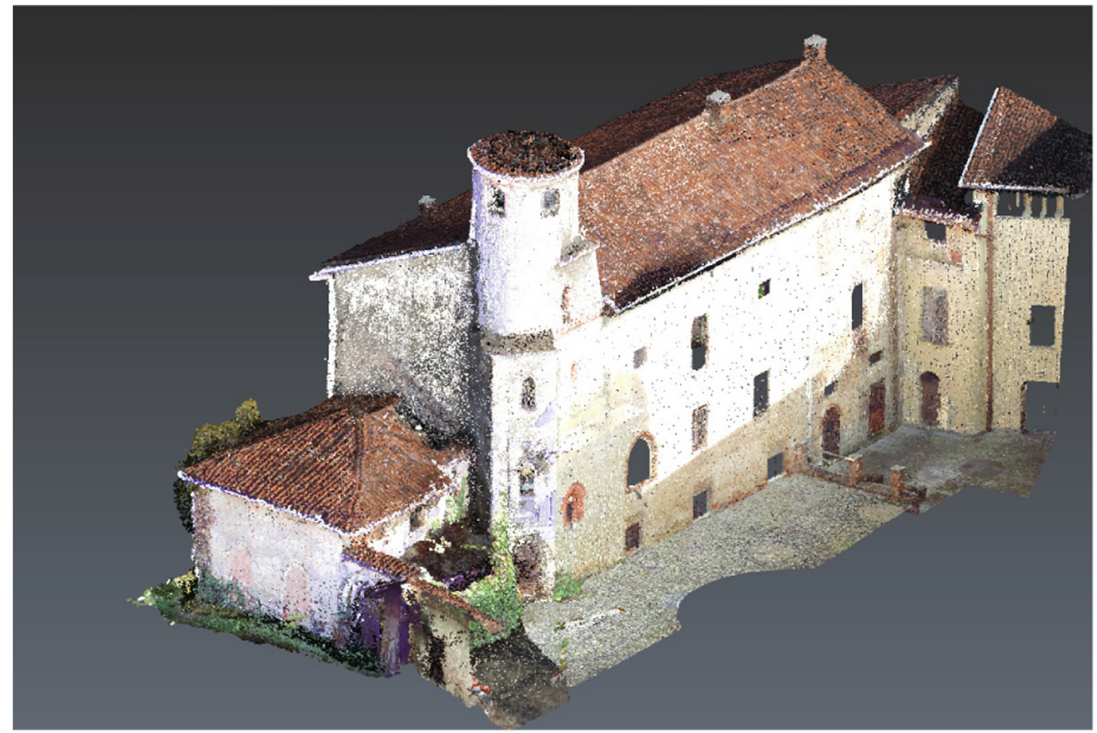

(a)

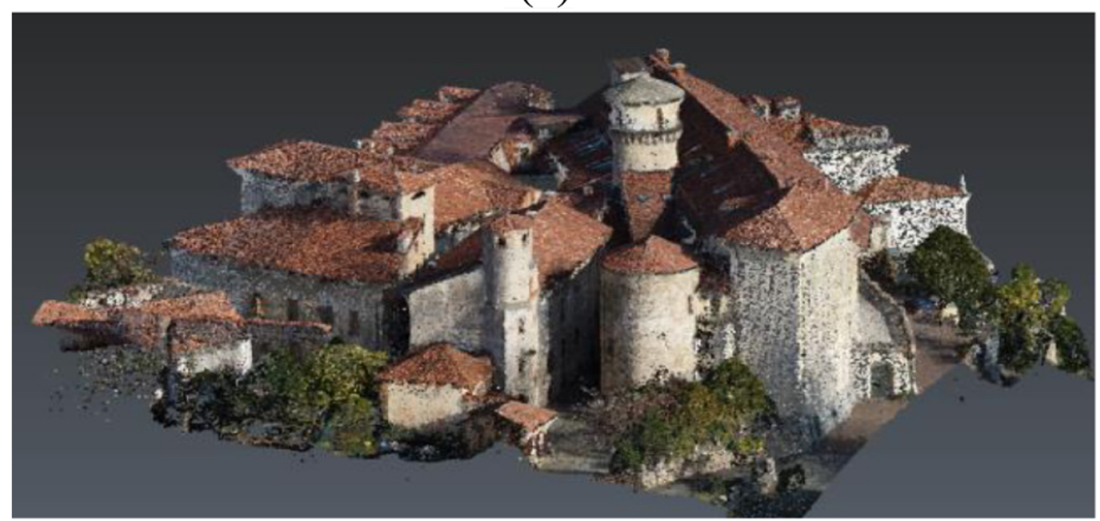

(b)
Fig. 6 Medieval mine Buca della Faina in San Silvestro archaeomining park (Brocchini et al. 2017)

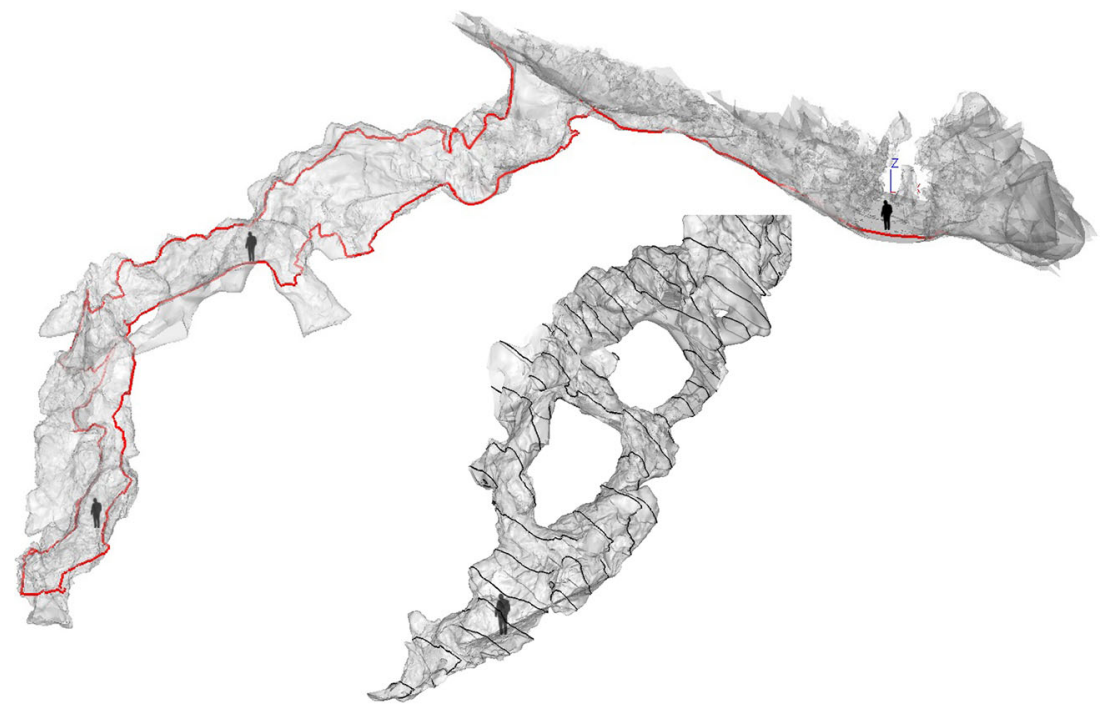




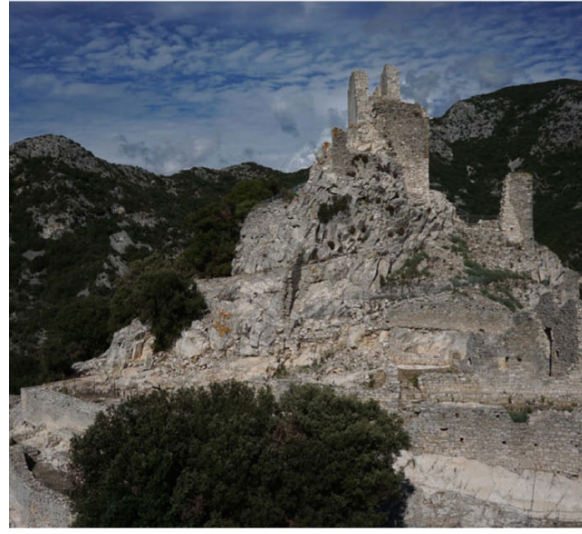

(a)

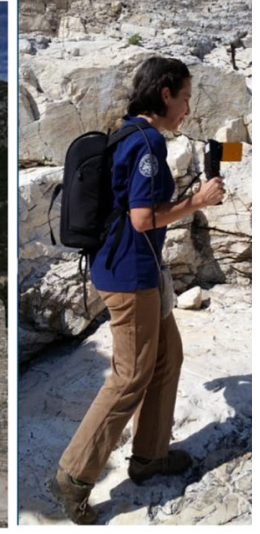

(b)

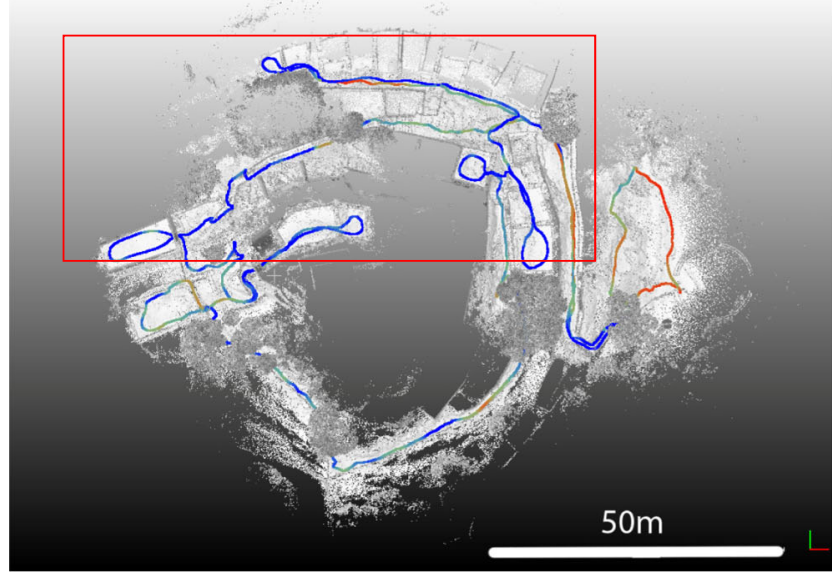

(c)

Fig. 7 The mapping of Rocca San Silvestro (a) by ZEB-REVO (b) in 2016, and the 2017 integration in rex box (c) (Brocchini et al. 2017)

- Trajectory and loops should be planned according to the local environment configuration. They are fundamental because the SLAM-based system is originated on the iterative alignment of extracted profiles that are based on that featuring attributes of the space. Indoor spaces or outdoor enclosed environments are favourable for better performance of the ZEB system. Roundtrips are preferred to avoid drift error propagation in "swing" effects or linear deviation.

- Time of acquisition is considered the sum of the few minutes required for initialisation and closure with an automatic in loco pre-processing and storage of data in the embedded memory and the trajectory execution.

Table 3 RMSE on the control points in the CRP model computed inside the tower of Valperga castle

Metric control residuals on the CRP model

\begin{tabular}{lllll}
\hline (m) & $X$ & $Y$ & $Z$ & Tot \\
\hline GCP & 0.004 & 0.003 & 0.004 & 0.007 \\
CP & 0.005 & 0.003 & 0.013 & 0.014 \\
\hline
\end{tabular}

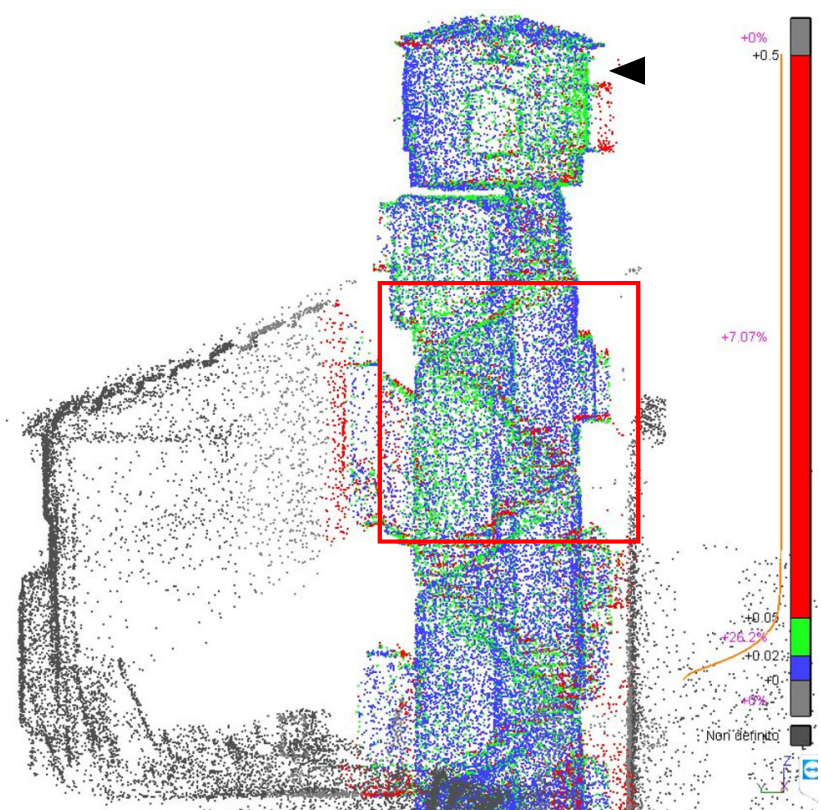

(a)

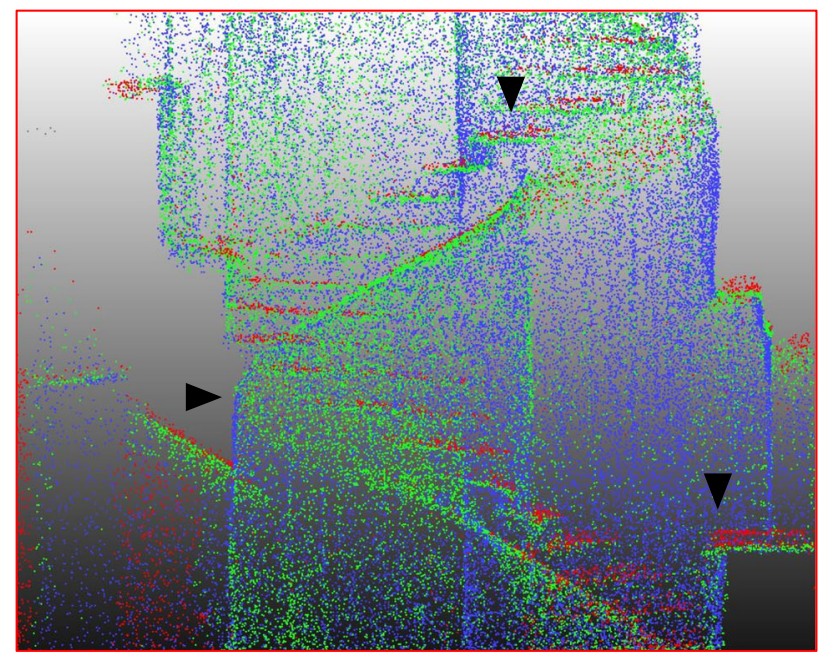

(b)

Fig. 8 Deviation errors in range colour map from the comparison between the full raw ZEB point cloud and the CRP reference model (a) and zoomed excerpt (b)

Considering an on-foot walking operator with a handheld device with a speed of almost $1-1.5 \mathrm{~m} / \mathrm{s}$, an itinerary of utmost 20-30 $\mathrm{min}$ is recommended for the best precision of outcome data.

Table 4 Statistical values of the deviation analysis of the separated O\&R paths for the cylindrical tower
Outward and return comparison on ZEB surface

\begin{tabular}{lll}
\hline & Raw & Optimised \\
\hline Mean & 0.091 & 0.021 \\
St.dev & 0.131 & 0.028 \\
\hline
\end{tabular}




\section{Experimental section: validation strategy}

The validation of the systems is always essential to determine how to use it in different application fields and determine the effectiveness of the systems based on the different contexts.

As is well known and has been already stated, the complexity of the documentation and modelling of $\mathrm{CH}$, as well as being motivated by physical reasons, is reflected in the uses and in the extreme variety of interdisciplinary acknowledgements and comparisons that the products must satisfy.

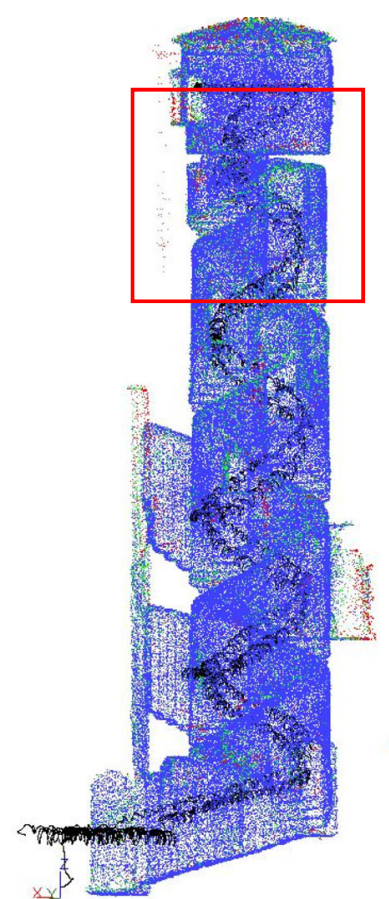

(a)

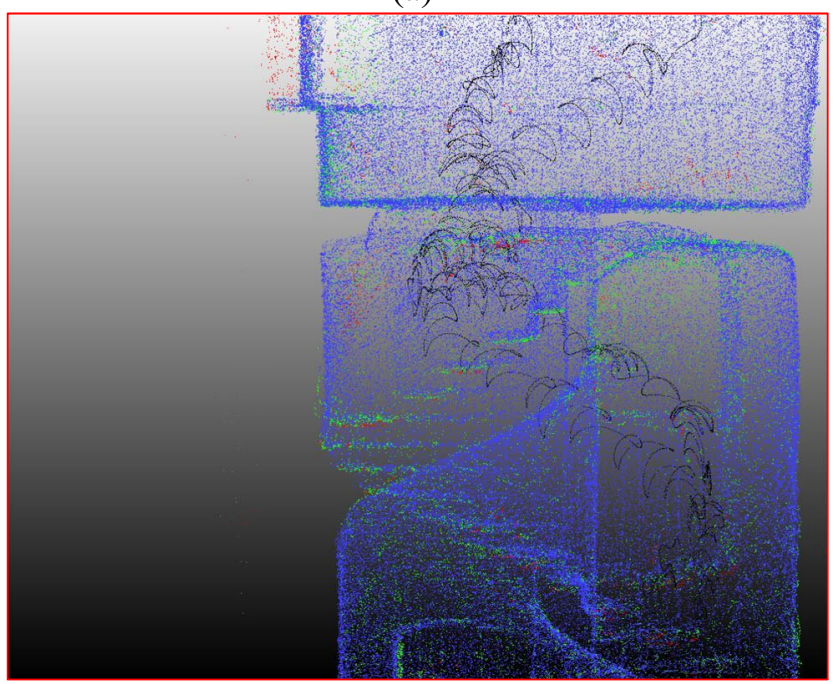

(b)

Fig. 9 Deviation error representation in range colours for outward and return ZEB point comparison (a) and zoomed excerpt (b)
Although the relationships between the future uses of point clouds/3D model and the validation strategy may not be straightforward, it is crucial to adopt validation rules involving metric quality and other parameters concerning the usability of datasets and to consider the application in a significant sampling that can cope with the uniqueness that the cultural complexes present.

In other words, the validation concept that verifies the specified requirements was assumed to be sufficient for the intended use (ISO/IEC Guide 99:2007 \& JCGM 200 2007).

To control the overall metric quality of the ZEB clouds, their reliability was firstly considered based on their single use. The first statistical parameter that contributes to the evaluation of the overall reliability was the accuracy, so some surfaces or clouds derived from more precise measurement systems were considered as ground truth to evaluate the deviation of the studied clouds by means of root mean square error (RMSE). It is also necessary to consider the precision of the clouds, because the precision is linked to the concept of repeatability of the measurements and is normally described by the standard deviation (St.dev) parameter. Thus, for the ZEB clouds, the intrinsic precision that is linked to the acquisition mode has always been considered.

With reference to the previous paragraph, the SLAM system, after calculating the raw trajectories, uses an iterative ICP-like process of automatic cloud-to-cloud profile registration to generate the $3 \mathrm{D}$ cloud, and this process has always been controlled using loop paths. In addition, the correspondence of the detected surfaces during the time acquisition has been carefully optimised in a non-automatic way by segmenting the clouds and supplementary cloud matching and optimisation operations. Moreover, even if the ZEB scanner system is provided for the indoor and outdoor environments, which has been studied in the aforementioned literature (Díaz-Vilariño et al. 2017; Nocerino et al. 2017; Thomson et al. 2013; Zlot et al. 2014), the level of accuracy and detail and the noisiness of the clouds are quite different. Therefore, the validation strategy in this work includes datasets acquired in different outdoor and indoor configurations to account for these differences.

Table 5 Statistical values of the deviation analysis of the O\&R paths with the reference CRP model for the cylindrical tower of the Valperga castle

Comparison of ZEB 3D data on ground truth

\begin{tabular}{lllllll}
\hline & Full raw & \multicolumn{2}{l}{ Raw } & & & \multicolumn{2}{l}{ Optimised } & \\
\cline { 3 - 4 } \cline { 6 - 7 } & Roundtrip & Outward & Return & & Outward & Return \\
\hline Mean & 0.025 & 0.026 & 0.024 & & 0.025 & 0.022 \\
St.dev & 0.034 & 0.034 & 0.032 & & 0.030 & 0.025 \\
\hline
\end{tabular}


Table 6 Statistical values of the deviation analysis of the separated paths O\&R for the ancient ice house of Valperga castle
Outward and return comparison on ZEB surface

\begin{tabular}{lll}
\hline & Raw & Optimised \\
\hline Mean & 0.078 & 0.047 \\
St.dev & 0.114 & 0.063 \\
\hline
\end{tabular}

Lastly, since the need to obtain multiscale and multicontent heritage models has been ascertained and shared, the possibility of using the ZEB scanner in multisensor surveying configurations has also been investigated. In the validation strategy for this study, the abilities of integration relating to the ZEB clouds were compared with other cloud surfaces acquired with systems that offer different resolutions and accuracies and provide therefore different scales of surveys.

In these cases, the dense clouds to which the ZEB data was integrated were mainly derived from UAV photogrammetry, and the DSM and other photogrammetric products were generated to document the overall set of sites or clouds derived from TLS or CRP. To obtain the reference clouds (using UAV photogrammetry, TLS, or CRP techniques), the usual criteria and pipelines were adopted, and they are not described in this paper. For the generation of photogrammetric clouds, the orientation of the blocks of images and the control of the results occurred through using GCPs (Ground Control Points) and CPs (Check Points), or in the LiDAR terrestrial applications, cloud recording with integrated cloud-to-cloud alignment techniques and references (again GCPs and CPs). "Test dataset presentation" provides the framework of the test datasets based on the selections related to the validation strategy.

\section{Test dataset presentation}

The datasets being validated belong to two projects of metric documentation of two vast cultural complexes belonging to different construction periods that can be ascribed to architectural heritage and the archaeological site.
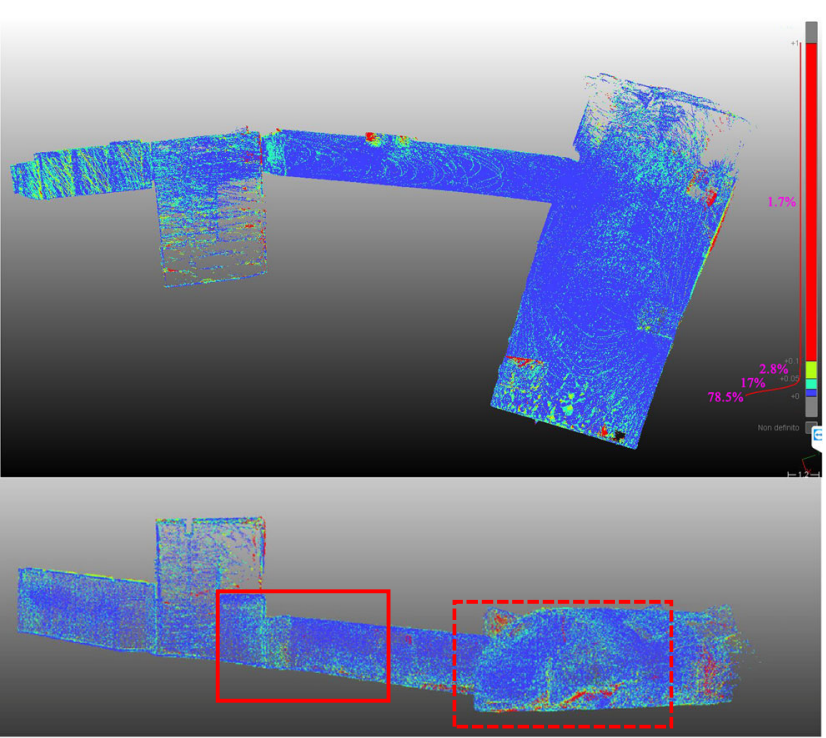

(a)

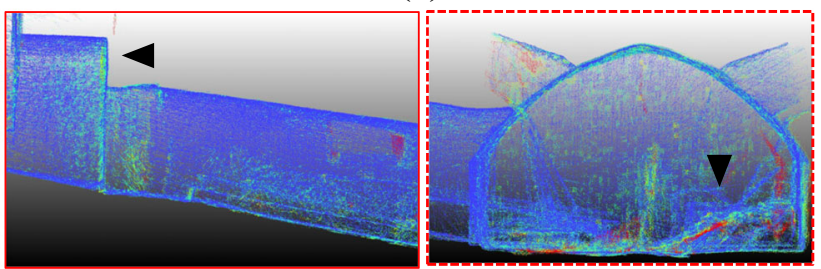

(b)

Fig. 11 Deviation error representation (top and lateral views) between optimised ZEB point clouds of O\&R in the ice house (scale ranges in Table 7). Top and side views (a) and zoomed excerpts (b)

The first site corresponds to the castle of Valperga (Turin) built in a strategic defensive position on the top of a hill and forming a system of palaces and gardens "at the French mode" between the 17th and 18th centuries (see also Chiabrando et al. 2017a, b). The second site is the San Silvestro fortress located in the homonymous archaeological park that includes a territory rich in mines frequented from the Etruscan age (VIII-I cent. BC) until the XX century. Both sites were subjected to a multisensor survey to obtain multiscale models

Fig. 10 Deviation error representation in range colours between raw ZEB point clouds of outward and return in the ice house (scale ranges in Table 7)

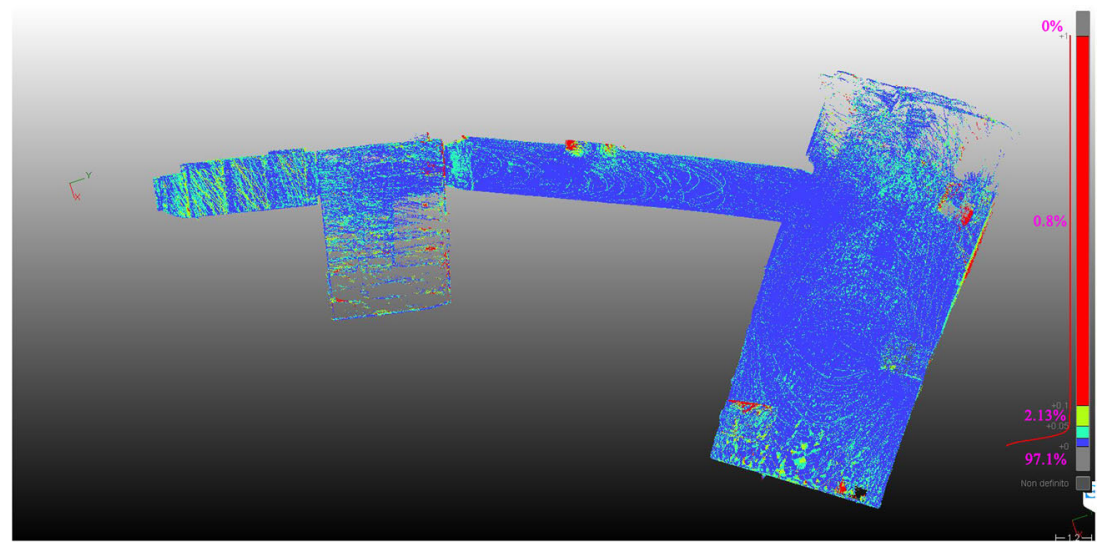


Table 7 Statistical results segmented in range error values from the deviation analysis of $\mathrm{O} \& \mathrm{R}$

Comparison errors in ZEB surface outward and return

\begin{tabular}{llll}
\hline & & Raw $(\%)$ & Optimised $(\%)$ \\
\hline $0.00<$ error $<0.02 \mathrm{~m}$ & Blue & 78.5 & 81 \\
$0.02<$ error $<0.05 \mathrm{~m}$ & Cyan & 17 & 16 \\
$0.05<$ error $<0.10 \mathrm{~m}$ & Green & 2.8 & 2.2 \\
$0.10<$ error $<1.00 \mathrm{~m}$ & Red & 1.7 & 0.8 \\
\hline
\end{tabular}

derived from terrestrial and aerial techniques and formed by the integration of datasets at different resolutions and scales.

The sites can be read in the first column of Table 2, while the second and third columns state whether the recorded environments were indoor or outdoor. The (A), (B), and (D) test datasets regarding the inside of a cylindrical tower, an underground ice house, and a mining cave were evaluated using the stand-alone validation. The ZEB point clouds recording the courtyard in Valperga castle (C) and the dataset covering the inside paths of the Rocca of San Silvestro (E) were validated instead in their integration of the whole 3D models of the sites involving UAV, TLS, and CRP clouds.

\section{Tower (A)}

The first dataset considered was the cloud acquired along the narrow and restricted spiral staircase that runs inside a cylindrical tower of the castle of Valperga (Fig. 3a). The ZEB1 acquisition was performed starting from the outside and covering the stairway up to the dovecote and back to the entrance on the ground floor. (ZEB1 dataset 19,000,000 pts./10-min time acquisition).

The structural and material degradation of the staircase and the helical vault of marked constructive interest had already been detected by means of a CRP survey and 3D modelling techniques from the structure-from-motion (SfM) algorithms (Chiabrando et al. 2017a, b). This 1-cm accuracy point cloud has been the reference for evaluating the SLAM-based dataset.

\section{Ice cellar (B)}

Historical buildings often contain surprises; from an entrance of one of the buildings surveyed at the architectural scale in the Valperga complex, a long and dark corridor starts and goes under two blocks of the castle, gradually descending and leading to the ice cellar. This path and this buried space seemed excellent to challenge the potential of the SLAM-based ZEB system. Also, in this case, the acquisition using the handheld ZEB scanner was performed by completing the roundtrip, starting from the courtyard (Fig. 4b; ZEB1 dataset $13,900,000$ pts./6 min).

\section{Courtyard (C)}

The integrated image and range-based survey at Valperga castle was planned with the aim of merging the DSM derived from UAV photogrammetry computed by nadir and oblique images (Fig. 5b), with the dense and very accurate models of the TLS technique by the FARO Focus 3D X120 scanner (Fig. 5a). The use of the MMS ZEB scanner provided the opportunity to evaluate the use in such cases of buildings so densely packed with narrow courtyards that are not suitable for photogrammetric surveys of facades. In addition, the use of the scanner could avoid the heaviness and density in the usage of terrestrial LiDAR clouds. This was an opportunity then to evaluate the use of ZEB clouds in a relevant multisensor survey context. (Fig. 2; ZEB1 dataset 8,200,000 pts./4 min).
Fig. 12 Range colours representing elevation on the surface topography by ZEBREVO, with human size, from the street level (right), up to the entrance (black arrow, $+5 \mathrm{~m}$, red), down to the lower level (blue, almost $-17 \mathrm{~m}$ )

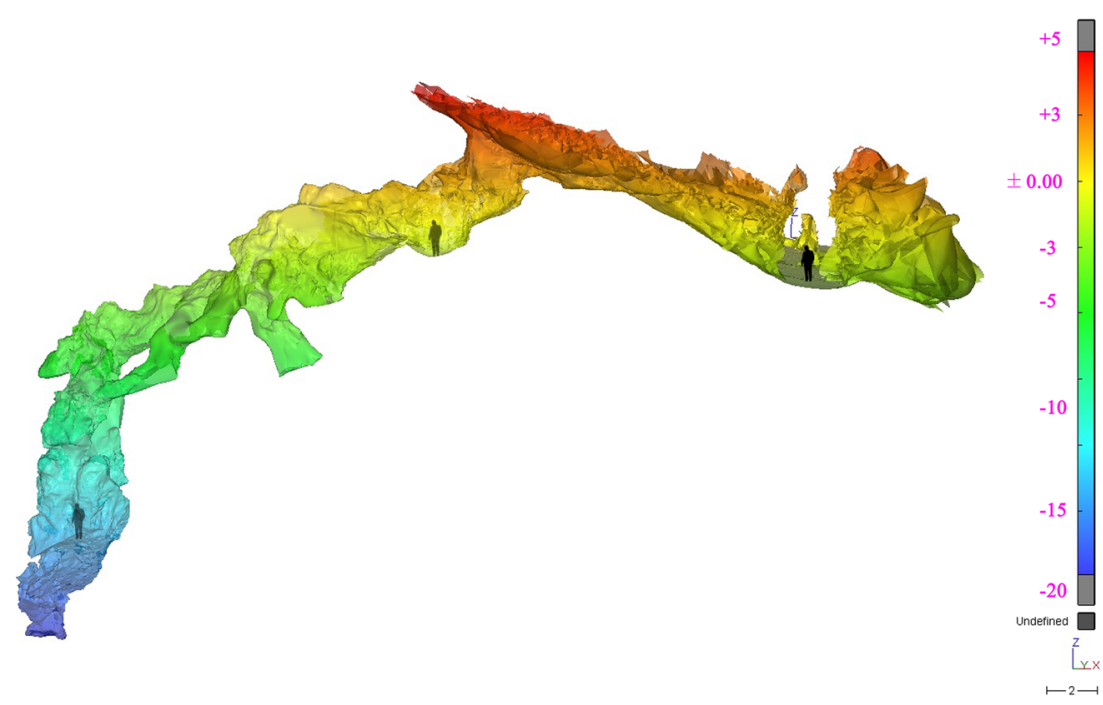


Fig. 13 O\&R raw point clouds refer to the Buca della Faina medieval mine and stress, in the excerpt, the closure error. The entrance location is represented by the black arrow

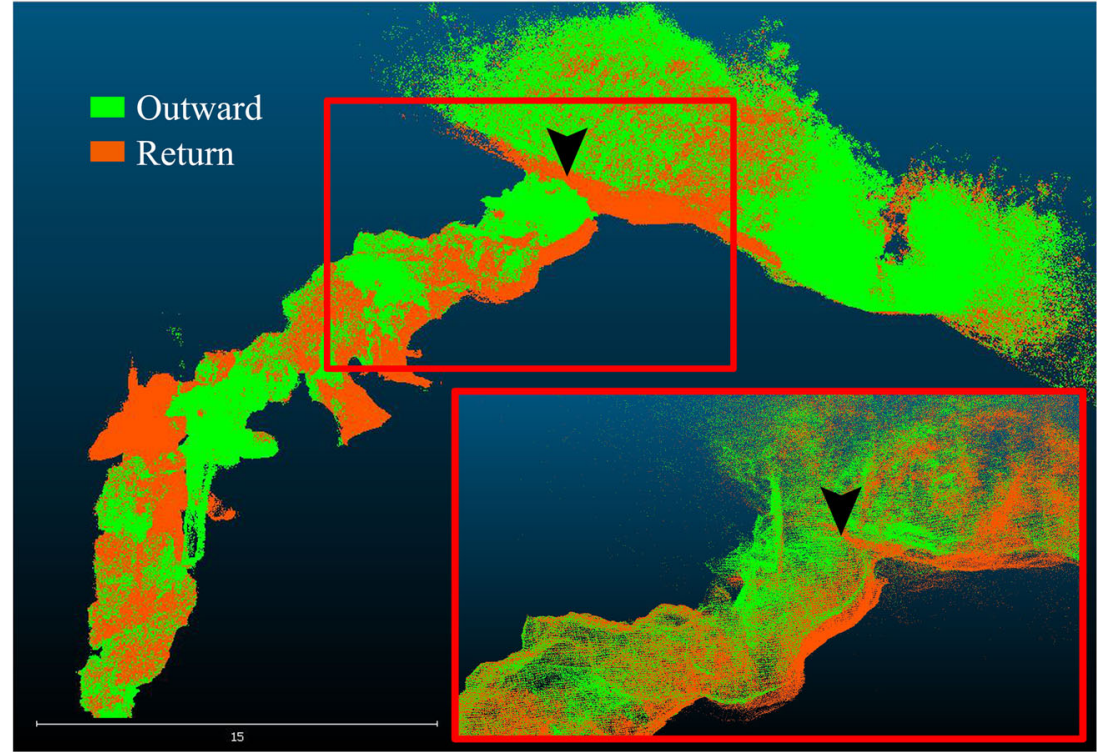

\section{Mining cave (D)}

Having ascertained that the handheld ZEB system is profitable for the modelling of underground environments such as quarries and mines, the scanner was tested in the medieval mine called Buca della Faina (Fig. 6; ZEB-REVO dataset 41,700,000 pts./ $25 \mathrm{~min}$ ).

This mine is strange because it can be traversed in many places only on all fours, and the cloud was only collected with the help of speleologists. The slowness of acquisition explains the point density of this cloud, which counts 42 million points compared to 33 million of the cloud acquired in the fortress $(\mathrm{E})$.

\section{Fortified village (E)}

Even the multistratified site of San Silvestro, with its safeguarding landscape and archaeological heritage, was subject to UAV and TLS acquisition and modelling. The densely built area needed detailed 3D LiDAR survey and models because it is subject to consolidation and restoration works since it lies on a slope of the hill presenting landslides.

The TLS survey, operated with a FARO Focus 3D X120 scanner, for the study of construction systems of masonries was

Table 8 Statistical values of the O\&R comparison subjected to the optimisation process

Outward and return comparison on ZEB surface

\begin{tabular}{lllll}
\hline & Raw & \multicolumn{2}{l}{ Optimising steps } & \\
\cline { 3 - 5 } & & Cleaning in/out & Filtering in/out & In only \\
\hline Mean & 0.214 & 0.175 & 0.103 & 0.055 \\
St.dev & 0.313 & 0.183 & 0.131 & 0.070 \\
\hline
\end{tabular}

necessarily heavy and time-consuming and was therefore an ideal site to test the validity of the clouds acquired by the ZEB system.

The loop acquisition was laid along the entire visit path of the fortress, starting from the entrance to the east and following exactly the ancient ascent to the culminating part of the fortified village and rearing from the other side according to the ancient road that embraces the cone-shaped Rocca.

In 2016, a ZEB-REVO dataset was collected along the whole circular pathway around the Rocca $(33,900,000$ pts./ $23 \mathrm{~min})$. In 2017, a second dataset (7,200,000 pts./8 min) was conceived as an integration of the area and is shown in Fig. 7 with an outward and return track.

\section{Metric validation in stand-alone solution}

A stand-alone use of point clouds derived by this SLAM-based mobile mapping is conceivable due to the intrinsic metric values of the endorsed concept of the so-called 1:1 scale of ranging measurements returning, and this method is helpful in mapping indoor volumes wherever georeferencing issues are not required. Once known and admitted as acceptable, the reliability verified on the reference model for (A) (the designated CRP model for the indoor space of the tower), the confidence level of the ZEB

Table 9 Statistical values of deviation errors grouped into ranges related to the main optimisation steps

Outward and return comparison errors in ZEB surface

\begin{tabular}{llll}
\hline & Raw $(\%)$ & Filtering $(\%)$ & In solo $(\%)$ \\
\hline $0.00<$ error $<0.10 \mathrm{~m}$ & 69 & 87.4 & 88.5 \\
$0.10<$ error $<0.20 \mathrm{~m}$ & 22.7 & 4.5 & 4.3 \\
$0.20<$ error $<0.6 \mathrm{~m}$ & 8.3 & 8.1 & 7.2 \\
\hline
\end{tabular}


Fig. 14 Range colour representation of deviation analysis errors for the only internal point cloud (scale ranges in Table 9)

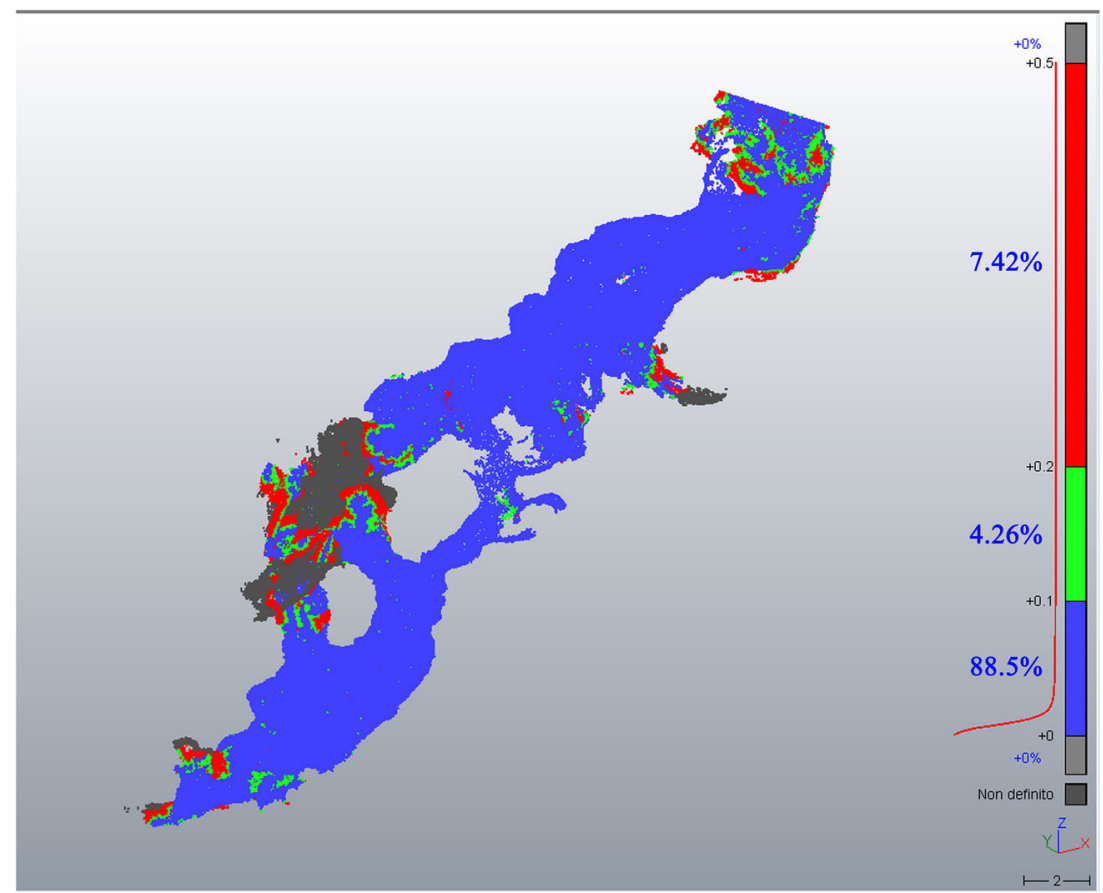

reconstruction in such indoor scenarios such as the ancient ice house (B) and the archaeomining cave (D) can be reasonably circumscribed and validated for a related scale use.

The problems about the establishment of the relationship with another reference surface are those problems that are primarily necessary to face because of the already clarified non-existence of positioning data and the lack of direct radiometric content in the raw data. Some referencing issues emerge in the operative fulfilment of this purpose. A first solution can be the matching of tie points that are detectable targets on both the 3D point cloud with $x, y$, and $z$ coordinates measured as references. Complications deriving from this discrete method include the difficulty in recognising and choosing the exact point; however, a statistical evaluation by means of RMSE on many matched point distances can grant the accuracy assessment (Farella et al. 2016) of the action. A more effective solution of point cloud alignment is commonly offered by the control of deviation errors on the performance of an ICP-like algorithm, a so-called cloudto-cloud, between the ZEB surface on the other point clouds (TLS; CRP). This method, more continuous along the whole considered surface, undergoes possible different precision of the surface characterisation in local details and suffers the

Table 10 RMSE on control points in the UAV photogrammetric reconstruction of Valperga castle

Metric control on the UAV photogrammetric DSM

\begin{tabular}{llllll}
\hline$(\mathrm{m})$ & $X$ & $Y$ & $Z$ & Plan & Tot \\
\hline GCP & 0.009 & 0.012 & 0.021 & 0.015 & 0.026 \\
CP & 0.012 & 0.009 & 0.025 & 0.014 & 0.029 \\
\hline
\end{tabular}

common noise factor in the ZEB surface. For these reasons, the alignment was evaluated without and after a process of optimisation (segmentation, outliers cleaning, noise filtering).

\section{The cylindrical tower (A)}

The CRP reconstruction inside the tower presented in Fig. $3 \mathrm{~b}$ is considered the ground-truth surface to validate the ZEB mobile mapping, and this surface has been computed with a controlled error propagation of less than $1 \mathrm{~cm}$ on GCPs and about $1.5 \mathrm{~cm}$ in the CPs (Table 3 ).

First, the cloud-to-cloud best fitting alignment on the CRP model of the full raw ZEB surface returned a first mean value of distance deviation of $0.025 \mathrm{~m}$ and a St.dev of $0.034 \mathrm{~m}$ (reported in the first column in Table 5), showing $67 \%$ of the points that actually deviated from the reference model of a value error $<$ $0.02 \mathrm{~m}$ and the $26 \%$ between $0.02<$ error $<0.05 \mathrm{~m}$ (Fig. $8 \mathrm{a}$ ).

Table 11 Metric control for the Valperga castle scans: the accuracy validation on the LiDAR point cloud registration shows a mean error of about $1 \mathrm{~cm}$ on target check points and a mean value of $4 \mathrm{~mm}$ on the clouds comparison

Metric control on the scans registration

\begin{tabular}{llllll}
\hline (mm) & Cloud-to-cloud alignment & & \multicolumn{2}{l}{$\begin{array}{l}\text { Residual error on } \\
\text { target points }\end{array}$} \\
\cline { 2 - 3 } \cline { 5 - 6 } & $\begin{array}{l}\text { Mean dev. } \\
\text { error }\end{array}$ & $\begin{array}{l}\text { Dev. error } \\
<4 \mathrm{~mm}\end{array}$ & & Mean & Max \\
\hline Courtyard & 3.35 & $55 \%$ & & 12 & 20 \\
\hline
\end{tabular}


Fig. 15 The courtyard wall surface in a ZEB profile reconstruction and $\mathbf{b}$ LiDAR DSM. c Deviation error representation in coloured range values, as stated in Table 12

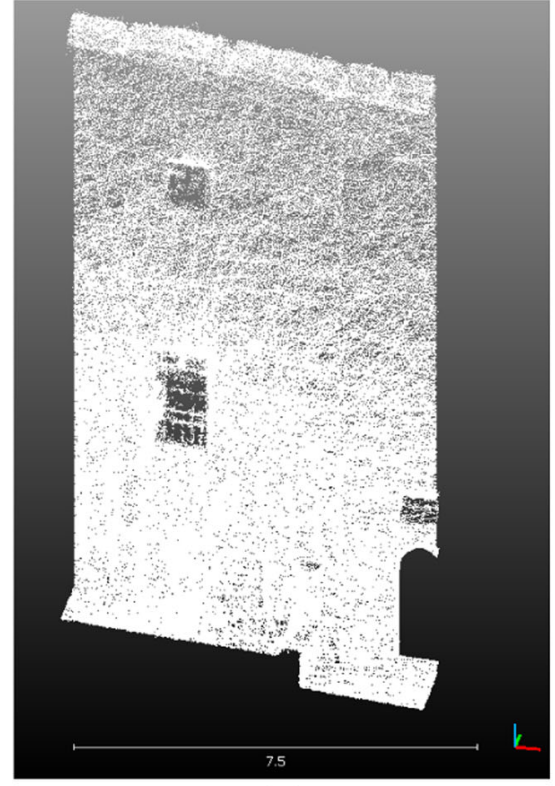

(a)

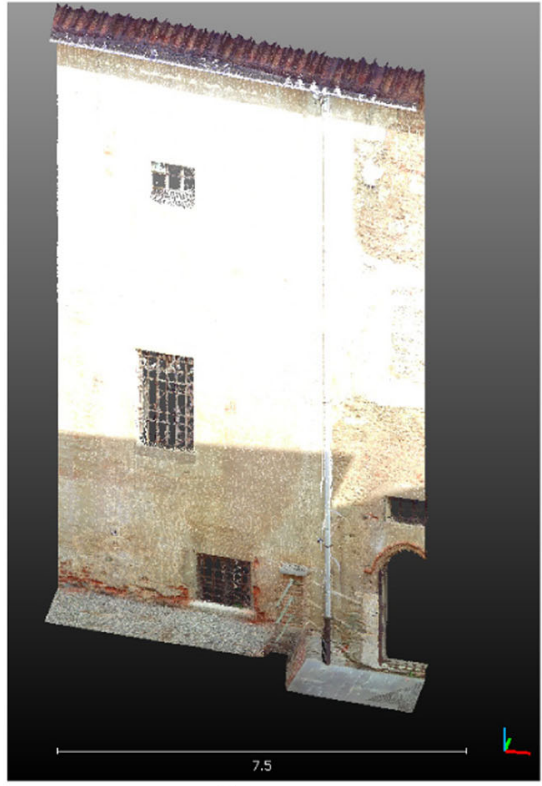

(b)

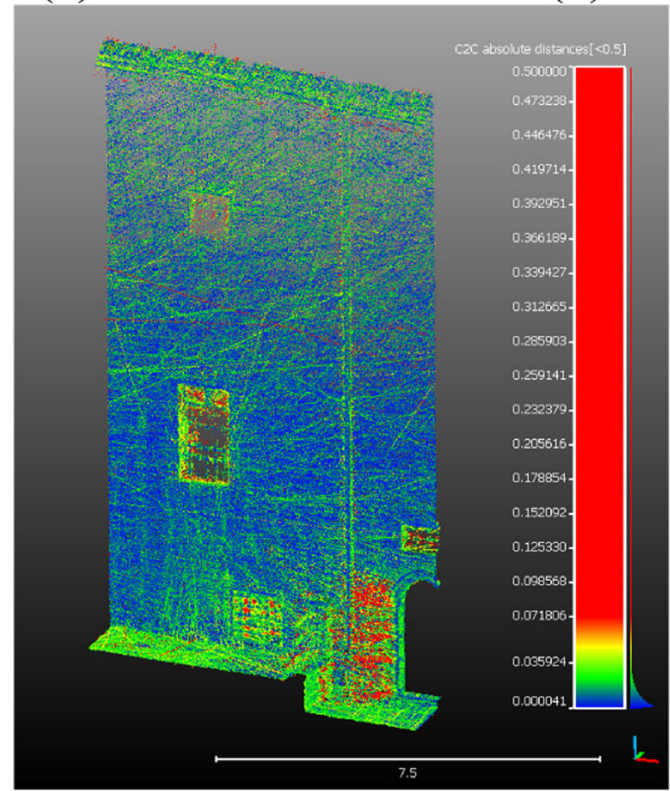

(c)

The most significant discrepancies $>5 \mathrm{~cm}$ are recognisable on the treads of the steps and in horizontal surfaces as windows and doors along the rising trajectory, as shown in Fig. 8b. In particular, the intermediate values of deviation, $2-5 \mathrm{~cm}$ in green colour, are spread in the spiral gradient of the stair ceiling and in the north side of the upper volume, as shown in Fig. 8a. The figure also shows the arrival of the outward trajectory as the farthest from the starting point of the scanning.

For these reasons, some crucial issues occur when the sequent validation is performed, separating the roundtrip and going into the ZEB cloud about its own precision evaluation, and these issues are strictly related to the precision of the operating principle on which the system is based. By separating the raw

roundtrip, which is helped by the time-marked trajectory, twofold clouds are obtained: the first trajectory, outward $(\mathrm{O})$, and the second trajectory, the return (R), take 6 min plus $4 \mathrm{~min}$,

Table 12 Statistical values divided into errors ranges corresponding to Fig. 15c
Comparison errors between ZEB and LiDAR surfaces

\begin{tabular}{ll}
\hline Mean & $0.017 \mathrm{~m}$ \\
St.dev & $0.023 \mathrm{~m}$ \\
$0.00<$ error $<0.02 \mathrm{~m}$ & $88.8 \%$ \\
$0.10<$ error $<0.05 \mathrm{~m}$ & $10.6 \%$ \\
$0.05<$ error $<0.20 \mathrm{~m}$ & $0.63 \%$ \\
\hline
\end{tabular}




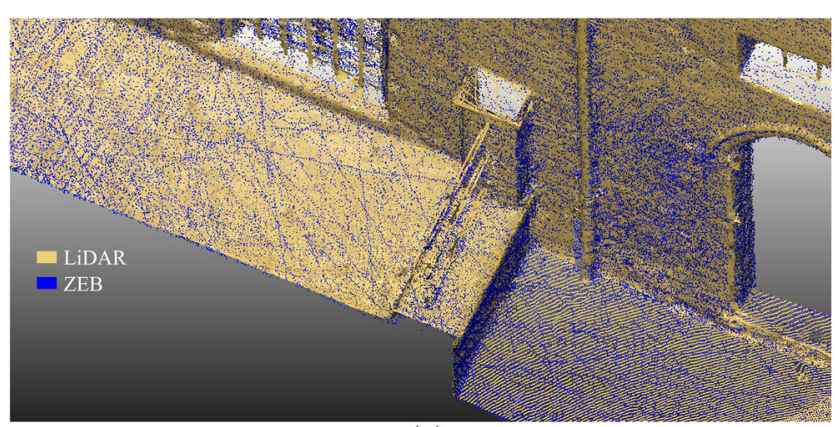

(a)

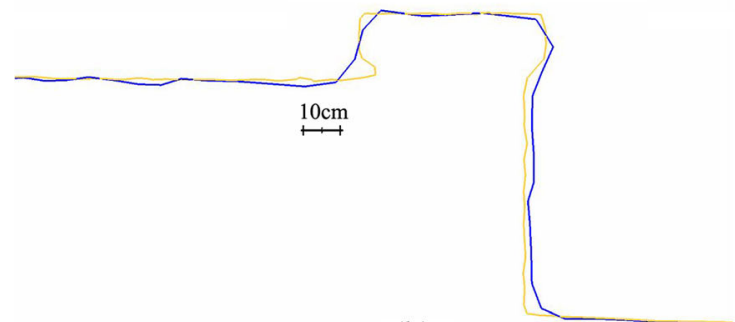

(b)

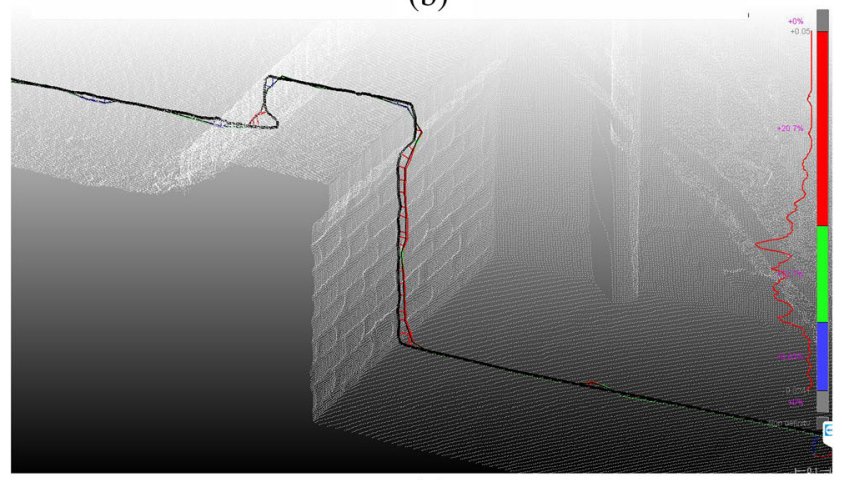

(c)

Fig. 16 The compared metric surfaces: ZEB1, blue, and TLS, gold (a). The extracted profile comparison on the courtyard pavement in the railing step (b). Thick stroke for the TLS profile, thinner for ZEB, and deviation error in range colours (c) respectively $(11,300,000+7,700,000$ points $)$. The relative alignment comparison, as listed in Table 4, retrieves critical values if the validation is performed without the cited optimisation of the surface points, denouncing a moderate problem of the SLAMbased alignment between outward and return (O\&R). For this type of path, Fig. 3a shows the coupling of the alignment with the noisy effect of the raw point cloud in outlier errors, affecting higher values.

An effective optimisation operation, as previously cited, on both O\&R provided the more suitable values in Table 4, and these values are provided in Fig. 9 and ensure greater reliability of the SLAM-based profile alignment solution implemented in the ZEB1. The reliability can be strengthened in the observation along the whole tower elevation in Fig. 9a and in the zoomed excerpt in $b$, where the figure shows the reduced deviation between the O\&R mainly bordered in the steps and in the roofing intrados. Deviation distance errors between O\&R are statistically represented with a mean value of $2 \mathrm{~cm}$ and a St.dev of approximately $3 \mathrm{~cm}$. Specifically, $93 \%$ of the points appear with deviation error values under $2 \mathrm{~cm}$ (Fig. 9a); however, the $99.5 \%$ is under $5 \mathrm{~cm}$. This precision proof cannot be admitted in a 1:50/ 1:100 scale of representation.

Furthermore, a second validation with the reference CRP model is thus consequently proposed: after both the separation values in O\&R and before and after optimisation execution values are presented in Table 5. Flanking is presented in the first column, and the initial values relate to the introduced full raw comparison.

The Table 5 values of comparison with the reference show the enhancing obtained in the discrepancies by optimisation operation applied on the O\&R ZEB surfaces, in which the discrepancies are affected by outliers and noise errors. The values in Table 5 display, however, a not very remarkable improvement in deviation values from the CRP reference
Fig. 17 The complete registration of several ZEB point clouds on the reference LiDAR DSM. The ZEB initialisation point is identified as a red star. The other surface integrations of sensors (ZEB and $\mathrm{UAV}$ ) are indicated in $\mathrm{A}^{-\mathrm{A}^{\prime}}$ and $\mathrm{B}-\mathrm{B}^{\prime}$

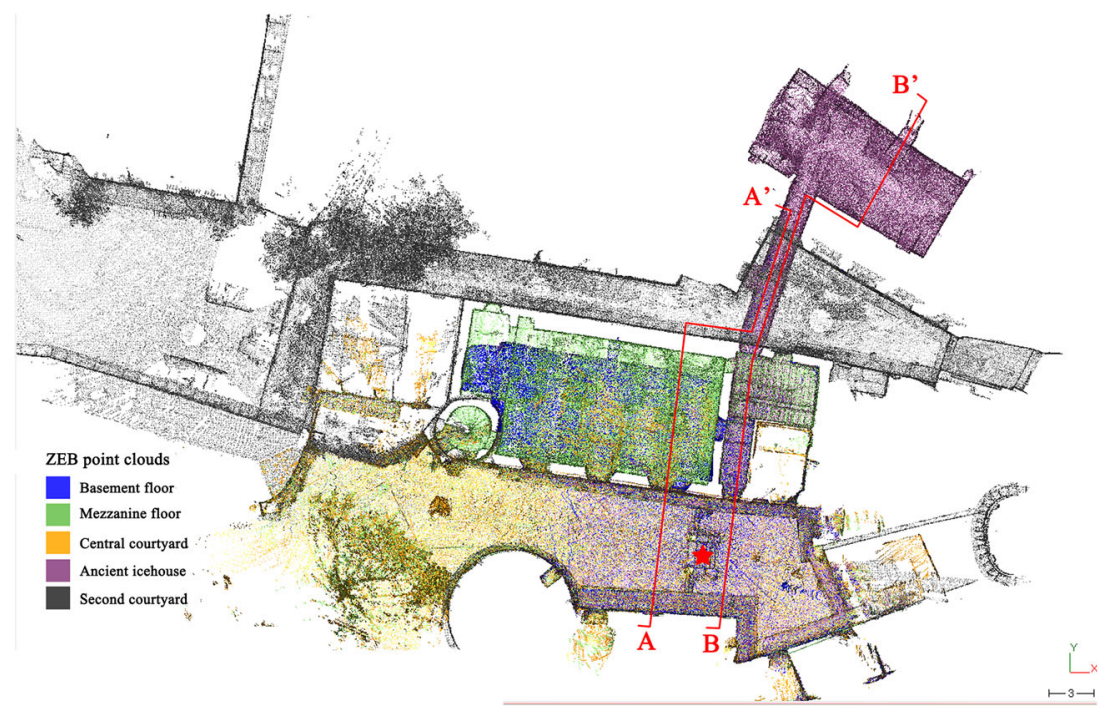


Table 13 Statistical values of the deviation between the ZEB and the TLS reference in the same courtyard

ZEB surface comparison with the LiDAR DSM

\begin{tabular}{lllll}
\hline & Courtyard & $\begin{array}{l}\text { Ancient ice } \\
\text { house }\end{array}$ & $\begin{array}{l}\text { Basement } \\
\text { floor }\end{array}$ & $\begin{array}{l}\text { Mezzanine } \\
\text { floor }\end{array}$ \\
\hline Mean & 0.065 & 0.057 & 0.052 & 0.053 \\
St.dev & 0.090 & 0.080 & 0.074 & 0.076 \\
\hline
\end{tabular}

model, proving the intrinsic accuracy. This improvement is limited to a mean of $2.2-2.5 \mathrm{~cm}$ with a St.dev of about $2.5-$ $3 \mathrm{~cm}$ for both the O\&R.

In particular, the outward values can be assimilated to those values representing the full raw comparison. Therefore, the return point cloud reconstruction based on the SLAM profile alignment approach actually benefits from the enclosed environment, and its reconstruction, as shown in the last column in Table 5, shows a better accuracy validation with a $2.2-\mathrm{cm}$ mean value from the reference photogrammetric model.

\section{The ancient ice house (B)}

According to the same principle of validation, in the (B) dataset, the verification of deviation error values has been compared before and after the optimisation approach, i.e. with the whole point cloud and surrounding, and then restricted to the
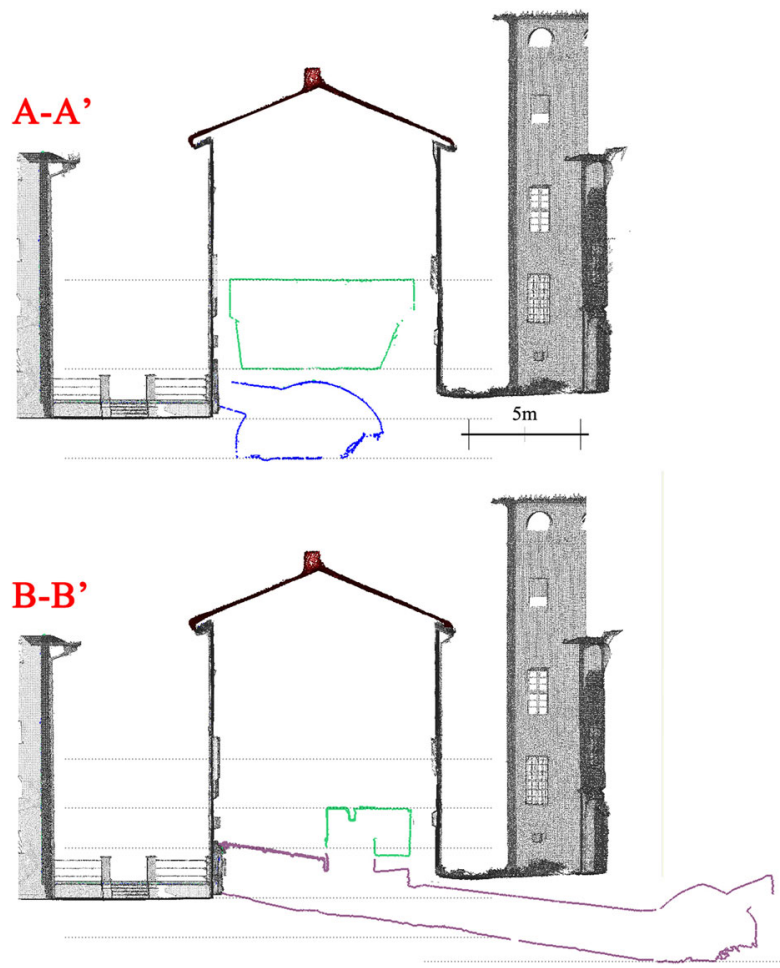

Fig. 18 Extracted sections, A-A' crossing the basement and mezzanine floors and $\mathrm{B}-\mathrm{B}^{\prime}$ crossing the entrance to the tunnel towards the ice house, connected with the volume in the mezzanine floor
Table 14 RMSE on control points in the UAV photogrammetric reconstruction of the Rocca

Metric control on the UAV photogrammetric DSM

\begin{tabular}{llllll}
\hline (m) & $X$ & $Y$ & $Z$ & Plan & Tot \\
\hline GCP & 0.012 & 0.017 & 0.024 & 0.021 & 0.032 \\
CP & 0.012 & 0.009 & 0.018 & 0.015 & 0.023 \\
\hline
\end{tabular}

underground path that leads to the ice house and to the mapping of the whole hypogeum volume.

The comparison between O\&R from the raw surface reconstruction that had the courtyard as a starting point (necessary to connect from a common area the whole 3D ZEB reconstruction, as the following presented) returned the subsequent values in Table 6. The deviation distances, as introduced, suffer from noise and outlier errors, and the optimised process leads to a reduction in discrepancies of approximately $50 \%$.

If deviation maps are examined, as shown in Fig. 10, to compare the raw O\&R point clouds, and in Fig. 11 for the optimised and localised surfaces, the establishment of range values of deviation errors distances, as reported in Table 7, allow the interpretation of some key aspects.

In Fig. 10, while in the arrival to the ice house, in the farthest point from the initialisation, the ZEB SLAM system aligned both the raw O\&R profiles with deviation errors referable to the blue colour for most of the hypogeum area, and the comparison of starting and arriving profiles in the courtyard significantly affected the statistical values, as shown in Tables 6 and 7. The optimisation operation, whose comparison errors are represented in Fig. 11 and listed in Table 7, replaced more significant values supporting the precision aptitude of the system in the 3D mapping of such narrow passages, but some critical areas remain in specific details, in particular, the values in green/red colours. These decreases of precision are identifiable, for example, in the ceiling of the starting portion of the passage towards the ice house, at the beginning of the scanning process; in some parts of the volume shown in Fig. 11a, in which these decreases are due to the space occupied by unrelated masses differently responding to the lightwave signal; in the ventilating outlet elements; and in some vertical planes (Fig. 11a) during the descending passage, which are differently modelled by the outward and return profiles

Table 15 Metric control on LiDAR scans in the Rocca

\begin{tabular}{|c|c|c|c|c|}
\hline \multicolumn{5}{|c|}{ Metric control on the scan registration } \\
\hline \multirow[t]{2}{*}{$(\mathrm{mm})$} & \multicolumn{2}{|c|}{ Cloud-to-cloud alignment } & \multicolumn{2}{|c|}{$\begin{array}{l}\text { Residual error on } \\
\text { target points }\end{array}$} \\
\hline & $\begin{array}{l}\text { Mean dev. } \\
\text { error }\end{array}$ & $\begin{array}{l}\text { Dev. error } \\
<4 \mathrm{~mm}\end{array}$ & Mean & Max \\
\hline Village & 4.58 & 50.8 & 18 & 35 \\
\hline
\end{tabular}


Fig. 19 Alignment strategies: a (I) ICP-like deviation error range values, explicated in Table 16; b (II) matching points, whose RMSE is also explicated in Table 16

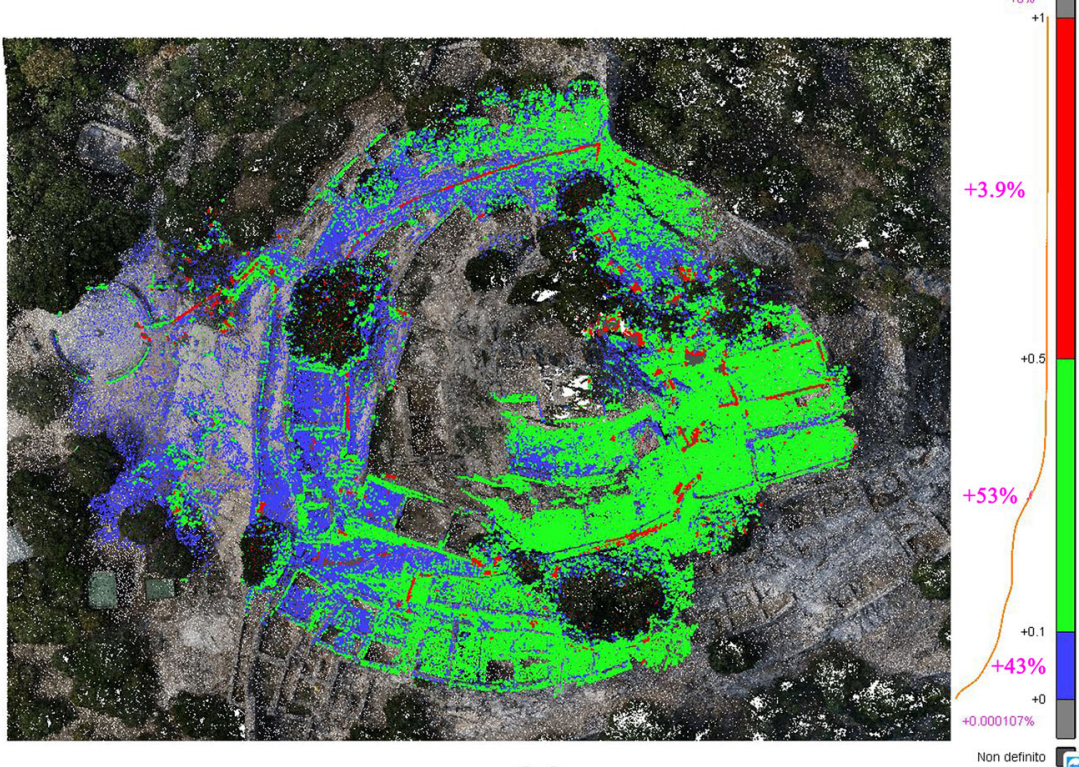

(a)
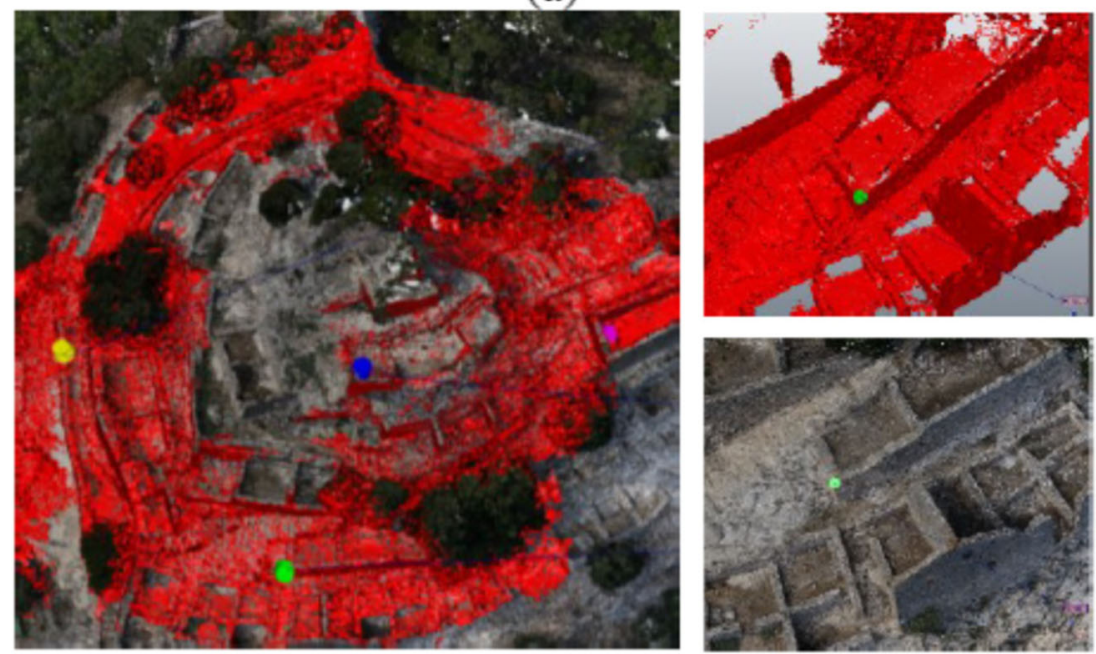

(b) possibly due to the topographic variation influence in the SLAM$3 \mathrm{D}$ reconstruction and due to the uniformity of some tunnel areas.

It is necessary to considerate, however, the blue-cyan colour profusion that is equivalent to a deviation error between $O \& R<$ $2 \mathrm{~cm}$ and $2 \mathrm{~cm}<\mathrm{err}<5 \mathrm{~cm}$. For the optimised surfaces, the deviation error corresponds to $81 \%$ of the points for the former and $16 \%$ for the latter, comprising an entirety of $97 \%$ of points that deviate in the global SLAM-based alignment of lower than $5 \mathrm{~cm}$.

\section{The mining cave (D)}

To validate the ZEB 3D mapping system in such a peculiar framework as the Buca della Faina sample shown in Fig. 6, the reasoning about the results should consider the local condition under which the acquisition has been performed, which is the very impressive number of points during the almost $26 \mathrm{~min}$ (nearly $1.7 \mathrm{million} / \mathrm{min}$ ) of the considerably limited trajectory length (only $\sim 100-\mathrm{m}$ roundtrip, almost $0.1 \mathrm{~m} / \mathrm{s}$ ) executed in a very intricate cave shown in Fig. 12, which featured limited accessibility for irregular topography, impervious spaces, significantly harsh surfaces, and reduced light conditions.

The verification of the O\&R deviation error on the ZEB points to validate and support the intrinsic precision of the SLAM-based operating system that underwent restrictive conditions was performed in two phases, before and after the optimisation. In this case, with the massive amount of irregular vegetation, it caused a noise error affecting the entrance zone and from the starting point to the arrival closure on the street level.

The first segmentation in O\&R parts consists of 14-min plus 12-min paths $(22,170,642+19,499,539$ points), from whose comparison a problem of loop closure occurred, as in Fig. 13, 
of almost $40 \mathrm{~cm}$. This loop closure distance result is within $8.3 \%$ of the point value, as shown in Table 9, meaning a mean deviation error between 0.20 and $0.60 \mathrm{~m}$. The deviation distribution along the whole path is, as shown in the first column of Table 8 , defined into $0.2 \mathrm{~m}$ with a St.dev of approximately $0.30 \mathrm{~m}$.

The high values are most likely conceivable because of the diffuse noise errors and of the weight in the statistical evaluation of the densely vegetated area at the entrance.

Based on these considerations, the validation approach tackled the possibility of properly segmenting the cloud and proceeding to the optimisation steps, dividing the data densification inside the mining cave at the entrance from the rest of the cloud outside. The two steps of cleaning and filtering are presented in the central columns of Table 8 and have been conducted parallelly to both segments, differentiating the parameter values and tailoring them according to the area (in, out). In the last step, the validation considers the deviation results only related to the $\mathrm{O} \& \mathrm{R}$ for the surface into the cave, and the deviation is presented in the final column of Table 8 .

Statistical results on deviation errors from the explained comparison have been grouped into simpler range values, in which the results are shown in Table 9, allowing better appreciation of the optimisation result improvement in the ZEB 3D reconstruction. Figure 14 presents the graphic representation of the in solo deviation analysis, whose values are reported in Table 9. The challenging 3D survey into the Buca della Faina reported a final evaluation on its own accuracy and established its confidence level for metric purposes: the obtained value is a mean error on O\&R based on the only cave of about $5.5 \mathrm{~cm}$ with a St.dev of $7 \mathrm{~cm}$.

\section{Metric validation in the multisensor survey}

The challenging aspects related to the use of this kind of spatial data, whose own precision has been investigated and accuracy evaluated in the previous section, are now faced based on its compatibly and integrability with other multisensory data on a multiscale survey organisation. The use of DSM coming from TLS with higher accuracy and UAV photogrammetry with significant 3D completeness and continuity is used in this phase and integrated to the ZEB reconstruction to evaluate their complementarity or exchangeability.

\section{The Valperga castle courtyard (C)}

The integration of the Valperga castle surveyed volumes is performed with LiDAR scans and UAV photogrammetric DSM.

The confidence level is based on the reliability of their metric reconstruction declared in Table 10 for the UAV photogrammetric reconstruction and Table 11 for the TLS and corresponded to an admitted scale of representation between $1: 50$ and $1: 100$.
Table 16 Statistical values for strategies I, absolute and divided in percentage of points per error ranges, and II, with residual deviation error on matching points

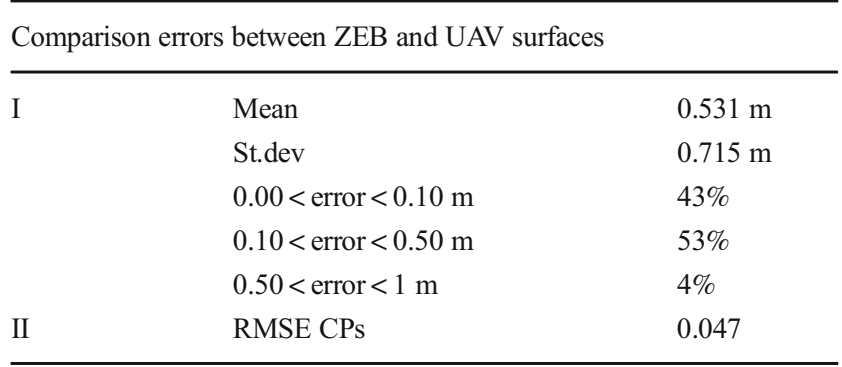

Two samples of $12 \times 7 \mathrm{~m}$ segments have been selected and optimised, as shown in Fig. 15a, b: 450,000 pts. for the ZEB segment and 5,600,000 for the LiDAR DSM, which is more than 12 times denser than the ZEB one. From the comparison analysis shown in Fig. 15c, the detected deviation errors with mean values of almost $2 \mathrm{~cm}$ ( $2.3 \mathrm{~cm} \mathrm{St.dev})$, as reported in Table 12, confirm the possibility to control the accuracy of the SLAM-based system in an outdoor scenario between a 1:100 and 1:200 scale of representation, in which $99 \%$ of points deviate from LiDAR less than $5 \mathrm{~cm}$.

The problem shown in Fig. 15c indicates the deviation (green) increasing with the height, which is due to a kind of systematic error related to the mechanical system of distribution mode of laser rays in ZEB1, as confirmed in Cadge (2016) (this has been improved in profile uniformity coverture by the ZEB-REVO system). However, the tangible limited descriptive capabilities intrinsic in the ZEB system, as expected, are localised in architectural details and edges, as shown in Fig. 16, and they become rounded and approximated. Analytical range values display

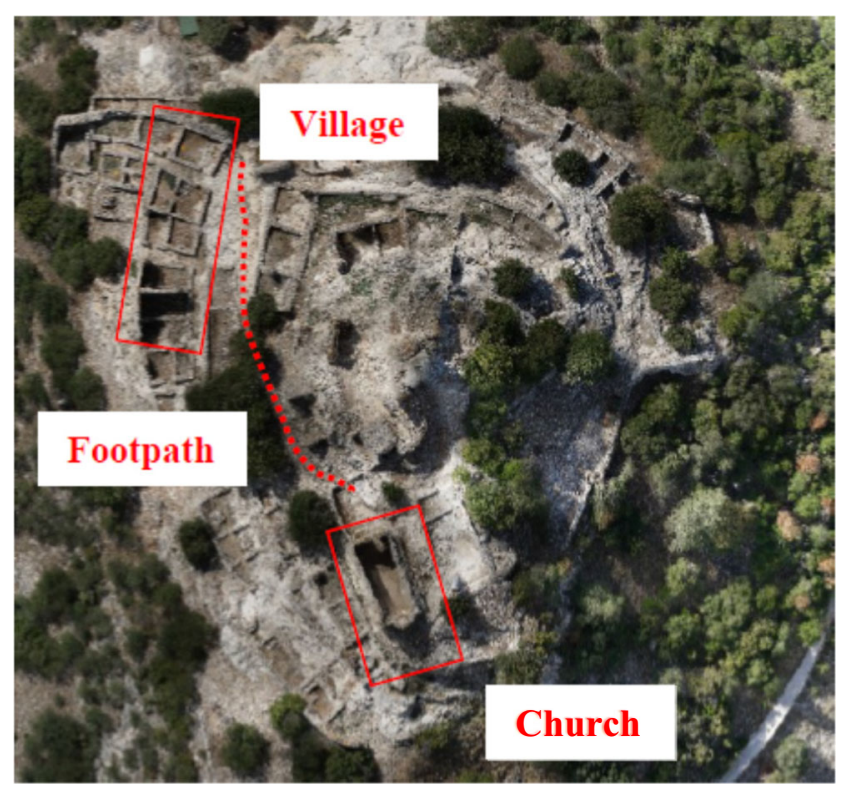

Fig. 20 UAV orthoimage of the San Silvestro Rocca with indications of the sample area in the lower village, the church, and the climbing path to the upper area 
Table 17 Church: statistical values for strategies I and II

Comparison errors between ZEB and UAV surfaces

\begin{tabular}{lll}
\hline I & Mean & $0.578 \mathrm{~m}$ \\
& St.dev & $0.771 \mathrm{~m}$ \\
II & RMSE CPs & 0.126 \\
\hline
\end{tabular}

errors of $\pm 1 \mathrm{~cm}$ (green) for $72.7 \%$, almost $20.7 \%$ for $+1 \mathrm{~cm}<$ error $<+2 \mathrm{~cm}$ and $6.3 \%$ for $-1<$ error $<-5 \mathrm{~cm}$.

In the proposed validation, the TLS DSM served as a basis for the configuration and referencing of a system of roundtrip ZEB scans starting from initialisation in the courtyard setting and directed to the several castle volumes in Fig. 17.

All the SLAM-based mappings belong to the LiDAR DSM as reference and deviation error values are presented in Table 13. These error values are related to the raw alignment via the cloudto-cloud method and initial control of deviation distances. With better control of error propagation in such a kind of articulated 3D mapping via optimisation procedures of the single clouds, as cited, the accuracy related to the 1:200 scale of documentation is a more than achievable and a very reliable goal for the ZEB system.

Starting from the integration of 3D data in this multiscale and multisource model, some schematic cut sections, as shown in Fig. 18, prove the possible integration with the aerial DSM by UAV photogrammetry.

These sections can show the added value of this measurement technology to effectively support the spatial analysis of architectural environments based on its verified reliability and scale accuracy and in relation to the more consolidated TLS approach.

\section{The fortified village (E)}

The validation performed in the second outdoor architectural complex adds more critical issues to the testing of the ZEB system in 2016 because the wide area on which the trajectory
Table 18 Village:

statistical values for surface comparison

has been conducted $\left(\sim 6000 \mathrm{~m}^{2}\right)$, and in the loop mode $(\sim$ $660 \mathrm{~m}$ ), i.e., the circular closed path, avoids the roundtrip intended because the return path is along the same outward path.

Due to the critical issues clarified, a second dataset in 2017 was collected in an $\sim 2000-\mathrm{m}^{2}$ area and in the roundtrip loop mode $(\sim 450 \mathrm{~m})$ Fig. 7.

The reference surfaces employed in this phase are the UAV photogrammetric DSM, whose planimetric and vertical reliable accuracy are reported in Table 14, and the LiDAR scans are restricted to the rooms at the entrance area of the village, whose metric control is reported in Table 15.

The problem of referencing and validating the ZEB scan laid on the entire area of the village was performed in a circular loop. First, Fig. 7 shows the quality-marked trajectory superimposed to the width of the SLAM-based point cloud. The best working areas are generally those areas that have been travelled twice.

If a deviation analysis is performed on the comprehensive highly detailed UAV DSM, as shown in Fig. 19, to validate the behaviour of the ZEB surface on such a kind of reliable reconstruction, a crucial problem emerged, as reported in the form of statistical analysis in Table 16. It is reasonably attributable to the drift error affecting the circular path mode in such an irregular topographic setting, where open spaces and narrow passages alternate, straining the SLAM-based alignment algorithm operation. In fact, the analysis of the whole fitting verification retrieved higher values distributed in the path. By choosing the correspondences of some recognisable matching
Fig. 21 Alignment strategies: (left) I, ICP-like with the entire ZEB point cloud; (right) II, local alignment of segmentation by matching points
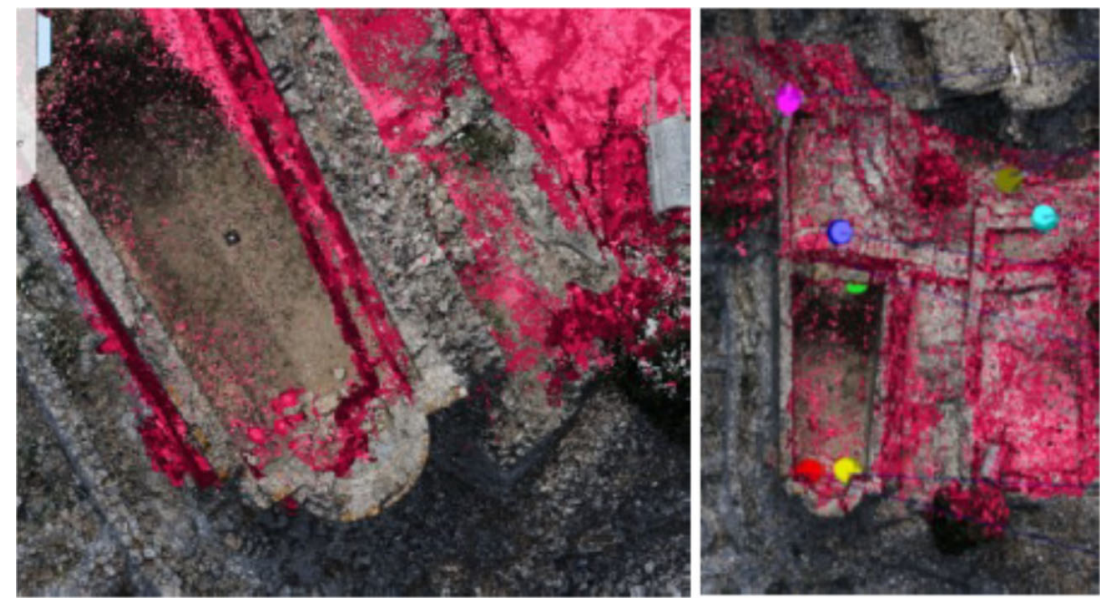
Table 19 Path: statistical values for surface comparison
Comparison between ZEB and UAV surfaces

\begin{tabular}{lll}
\hline Raw & Mean & 0.077 \\
& St.dev & 0.113 \\
Opt & Mean & 0.060 \\
& St.dev & 0.093 \\
\hline
\end{tabular}

points (i.e., corners) as the example of Fig. 19, the residual errors are locally reduced, as shown in Table 16, confirming the strong need of the system to base its operational effectiveness of featuring geometries in the environment, especially if an outdoor scenario is considered.

According to a profitable optimisation, the approach that can be deployed is based on filtering, segmenting, and separating non-improved parts, noising errors, and encumbering elements, such as rich vegetation inside the village.

The slight resulting variation obtained by this intermediate phase confirmed the intrinsic precision problem in the trajectory deviation, and a focused segmentation approach was performed to verify the drift areas and localise where the SLAM-based

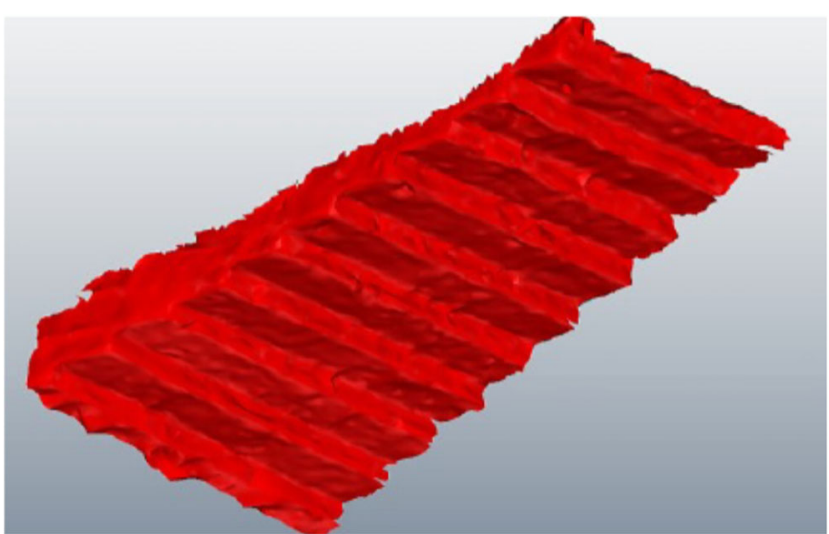

Fig. 23 Return of the achievable level of details in a sample triangulated mesh computed from the optimised ZEB surface

alignment underwent a linear deviation along the path in those cases where the trajectory quality denounced criticalities.

For example, in the higher area of the church, as identified in Fig. 20, the greater discrepancy is pinpointed with values that locally reach a mean value of almost $0.6 \mathrm{~m}$ with a St.dev of $0.7 \mathrm{~m}$, as shown in Table 17 . If the locally segmented surface is aligned by means of matching points in Fig. 21, the control on
Fig. 22 Comparison analysis between UAV DSM (blue) and ZEB DSM (red): a mapping of the discrepancies in two ranges 0 $5 \mathrm{~cm}$ and $5-10 \mathrm{~cm}$; b extracted section profiles describing steps

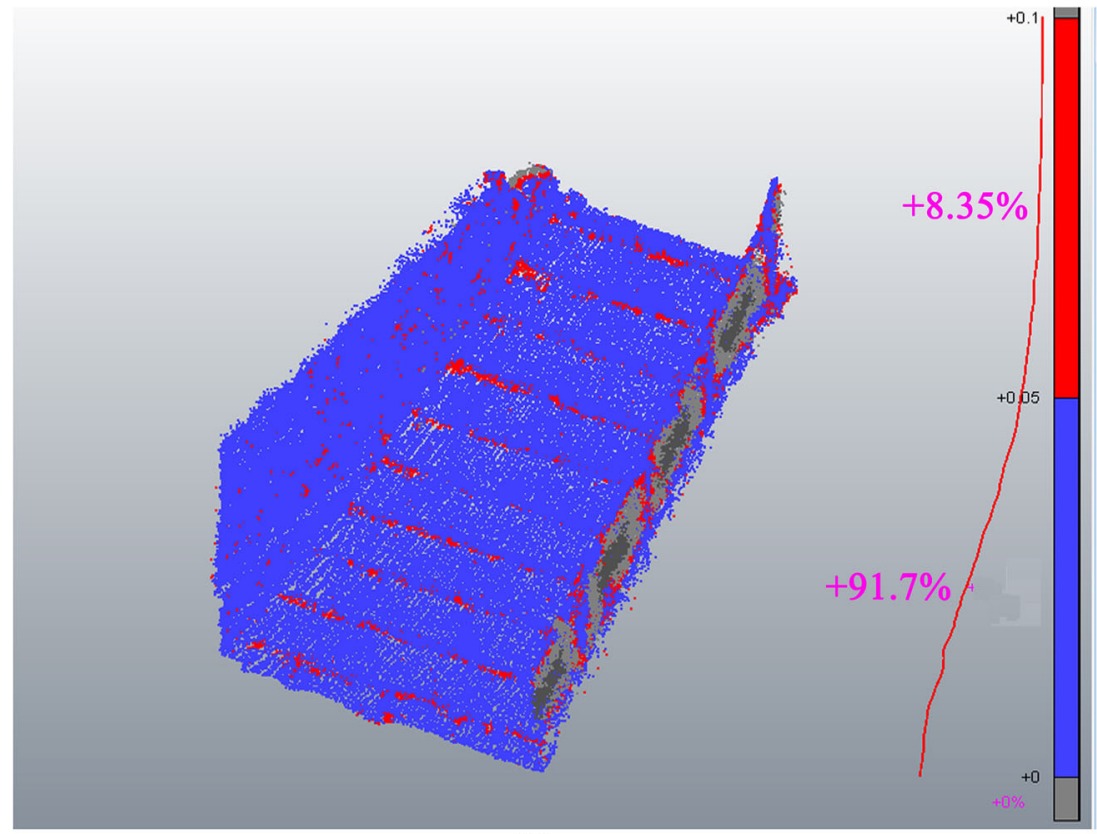

(a)

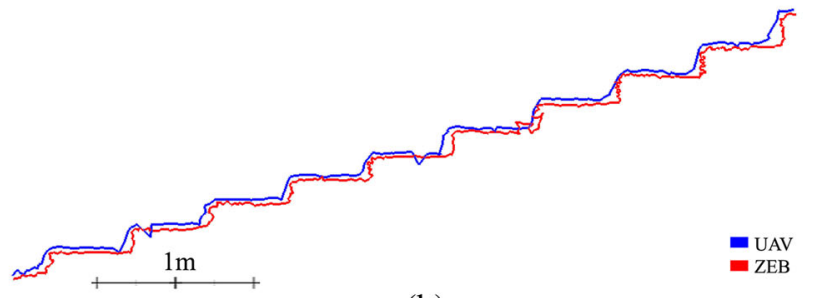

(b) 
residual errors on distances provides much better values, with an order of magnitude of $10 \mathrm{~cm}$, as shown in Table 17.

The segmented sample area in the lower village, as shown in Fig. 20, should be measured, and in this case, the 2017 ZEB 3D reconstruction was validated due to the beneficial O\&R trajectory mode employed for updating that area. By flanking the UAV DSM surface comparison, the deviation analysis with a LiDAR point cloud was computed with the TLS approach and added as a reference surface for the validation.

The residual values, which are summarised in Table 18, for this sample confirm the improved SLAM-based registration on the second dataset (2017), reasonably deviating more from the highly detailed TLS surface than the UAV model. This increase can be corroborated by the analysis and validation of the ZEB (2017) surface on the climbing path to the church. Table 19 reports the comparison values between ZEB and UAV DSM for the segmented narrow footpath made by steep steps, as shown in Fig. 22a, with lateral surfaces in a parallel analysis, pre-/postoptimisation. The analysis is finalised to evaluate, through their precision, the descriptive capabilities of both these rapid mapping approaches to replace a demanding TLS deployment.

If the segments of the stair samples are considered and both the contributions of the techniques are evaluated and compared, the ZEB reconstruction, as shown in Fig. 22b, potentially satisfies the scale detail of the mapping purposes from the ground, supporting and even perfecting the aerial photogrammetric purposes. Referring to the comparison of points shown in Fig. 22a, $0.00 \mathrm{~m}<91.7 \%$ pts. $<0.05 \mathrm{~m}$ and $0.05 \mathrm{~m}<8.3 \%$ pts. $<0.10 \mathrm{~m}$ (higher values are related, as visible, to the railing modelled by the ZEB and not reached by UAV).

The optimised ZEB surface can also profitably support the surface triangulation, as shown in Fig. 23, returning a remarkable level of detail, i.e., in step edges.

\section{Conclusion and future perspectives}

The cloud tests and analyses performed confirm an extremely positive judgement on the increased use of MMS GeoSLAM ZEB in the documentation of $\mathrm{CH}$ in the literature. Currently, the main problem in applying this technology is the lack of radiometric information that would seem to be the next update, starting with the recent introduction of a commercial off-the-shelf (COTS) camera whose images are not yet fully fused with the range data.

The actual benefits of the handheld system, flanking by critic points, can be summarised, and they are the following:

- Efficiency in indoor spaces and articulated architectural, landscape, and archaeological massings where GNSS positioning is not available.

- Rapidity in both acquisition and processing (this consideration is mainly addressed to the automatic pre-process of clouds, as seen when the marked time trajectory must be carefully controlled manually and when the essential aid of the colour ramp quality must be optimised).

- Compactness and handiness for unfriendly environments.

- Problems related to the lack of high-quality radiometric data. (The update started in 2017 with the equipment of a GoPro camera. The images can be projected on the surface points in post-processing, facilitating the point matching for scan registration, segmentation, and other analyses of the clouds).

- Demand of alignment verifications for loop configuration and closure.

- Need of validation in very high scale and small detail. At present, the scale of ZEB-derived models can be considered to correspond to what the tradition calls the architectural scale, that is, $1: 100$ or 1:200. For a full availability and handling of architectural details, a scale 1:50 or above is required, and more accurate clouds derived from the TLS technique continue to be needed.

By changing the perspective, this last point can show a new vision. The clouds derived from the roundtrip ZEB portable scans are lighter and easier to handle than those of the LiDAR technique, and in fact, this study shows how other authors prefigure a promising development perspective in the construction of 3D city models of historic cities and the possibility of facing big data problems.

In addition, the possibility of reducing the use of the timeconsuming LiDAR technique to only indispensable occasions has deep implications in the field of $\mathrm{CH}$.

As also emerged in discussions at conferences such as at the Geores2017 (Ottoni et al. 2017), the meaning of "monumental complexes" aims to identify a very wide range of characterisation of assets and actions of documentation projects. The characterisations can range from the physical complexity of the contexts, the extension and articulation of study objects, and immediately connect the concept of "complex sites".

Therefore, the need for multiscale studies, approaches that foresee the need for multidisciplinary interaction in studies and analyses, and outcomes that lead to the planning of conservation and restoration interventions articulated according to the various static-structural aspects, chemical materials, and innovations of materials must be considered. Particularly significant are the number of dedicated contributions, the themes of the use of HBIM platforms, and the contributions focusing the derivation from reality-based models of other models suitable for structural analyses in the static and dynamic field (Ottoni et al. 2017).

The request for multilevel modelling is an increasing issue; therefore, the studied technology that effectively fills the scales between the architectural detail and the building/urban scale enables intense and increasingly specialised uses to be prefigured. 


\section{Compliance with ethical standards}

Competing interests The authors declare that they have no competing interests.

Open Access This article is distributed under the terms of the Creative Commons Attribution 4.0 International License (http:// creativecommons.org/licenses/by/4.0/), which permits unrestricted use, distribution, and reproduction in any medium, provided you give appropriate credit to the original author(s) and the source, provide a link to the Creative Commons license, and indicate if changes were made.

\section{References}

Bauwens S, Bartholomeus H, Calders K, Lejeune P (2016) Forest inventory with terrestrial LiDAR: a comparison of static and hand-held mobile laser scanning. Forests 7(6):127. https://doi.org/10.3390/ f7060127

Bellekens B, Spruyt V, Weyn M (2014). A survey of rigid 3D pointcloud registration algorithms. AMBIENT 2014, The Fourth International Conference on Ambient Computing, Applications, Services and Technologies. 2014., (c), 8-13

Bosse M, Zlot R (2009). Continuous 3D scan-matching with a spinning 2D laser. In 2009 IEEE International Conference on Robotics and Automation (pp. 4312-4319). IEEE. https://doi.org/10.1109/ ROBOT.2009.5152851

Bosse M, Zlot R, Flick P (2012) Zebedee: design of a spring-mounted 3$\mathrm{D}$ range sensor with application to mobile mapping. IEEE Trans Robot 28(5):1104-1119. https://doi.org/10.1109/TRO.2012. 2200990

Brocchini D, Chiabrando F, Colucci E, Sammartano G, Spanò A, Losè LT, Villa A (2017). The geomatics contribution for the valorisation project in the Rocca of San Silvestro landscape site. In International Archives of the Photogrammetry, Remote Sensing and Spatial Information Sciences-ISPRS Archives (Vol. 42). https://doi.org/ 10.5194/isprs-Archives-XLII-5-W1-495-2017

Cadge S (2016). Welcome to the ZEB revolution. GEOmedia (3)22-26

Chiabrando F, Naretto M, Sammartano G, Sambuelli L, Spanò AT, Teppati Losè L (2017a). The 4Dilan project (4Th Dimension in Landscape and Artifacts Analyses). ISPRS-International Archives of the Photogrammetry, Remote Sensing and Spatial Information Sciences, XLII-5/W1, 227-234. https://doi.org/10.5194/isprsarchives-XLII-5-W1-227-2017

Chiabrando F, Spanò AT, Naretto M, Tocci C (2017b). Strati dei palinsesti e modellazione spaziale multi-scala: analisi integrate per la conservazione di sistemi culturali. In: ANANKE Speciale GEORES 2017, Altralinea, pp. 135-140

Dewez TJB, Plat E, Degas M, Richard T, Pannet P, Thuon Y, ... Lucas J (2016). Handheld mobile laser scanners Zeb-1 and Zeb-Revo to map an underground quarry and its above-ground surroundings. In $n d$ Virtual Geosciences Conference: VGC 2016. Bergen, Norway. Retrieved from https://hal-brgm.archives-ouvertes.fr/hal-01348956

Díaz-Vilariño L, Verbree E, Zlatanova S, Diakité A (2017). Indoor modelling from SLAM-based laser scanner: door detection to envelope reconstruction. ISPRS-International Archives of the Photogrammetry, Remote Sensing and Spatial Information Sciences, XLII-2/W7, 345-352. https://doi.org/10.5194/isprsarchives-XLII-2-W7-345-2017

Eyre M, Wetherelt A, Coggan J (2016) Evaluation of automated underground mapping solutions for mining and civil engineering applications. J Appl Remote Sens 10(4):46011. https://doi.org/10.1117/1. JRS.10.046011
Farella E, Menna F, Nocerino E, Morabito D, Remondino F, Campi M (2016). Knowledge and valorization of historical sites through 3d documentation and modeling. ISPRS - International Archives of the Photogrammetry, Remote Sensing and Spatial Information Sciences, XLI-B5, 255-262. https://doi.org/10.5194/isprsarchivesXLI-B5-255-2016

GeoSLAM CSIRO research (2016) http://geoslam.com/slam/csiroresearch/

ISO/IEC Guide 99:2007, JCGM 200 (2007) International vocabulary of metrology — basic and general concepts and associated terms (VIM). Available at: http://www.iso.org/sites/JCGM/VIMintroduction.htm

Lingua A, Noardo F, Spanò AT, Sanna S, Matrone F (2017). 3D model generation using oblique images acquired by UAV. ISPRSInternational Archives of the Photogrammetry, Remote Sensing and Spatial Information Sciences, XLII-4/W2, 107-115. https://doi. org/10.5194/isprs-archives-XLII-4-W2-107-2017

Mandelli A, Fassi F, Perfetti L, Polari C (2017). Testing different survey techniques to model architectonic narrow spaces. ISPRSInternational Archives of the Photogrammetry, Remote Sensing and Spatial Information Sciences, XLII-2/W5, 505-511. https://doi. org/10.5194/isprs-archives-XLII-2-W5-505-2017

Murtiyoso A, Koehl M, Grussenmeyer P, Freville T (2017). Acquisition and processing protocols for UAV IMAGES: 3D modeling of historical buildings using photogrammetry. ISPRS Annals of Photogrammetry, Remote Sensing and Spatial Information Sciences, IV-2/W2, 163-170. https://doi.org/10.5194/isprs-annalsIV-2-W2-163-2017

Nikoohemat S, Peter M, Oude Elberink S, Vosselman G (2017). Exploiting indoor mobile laser scanner trajectories for semantic interpretation of point clouds. ISPRS Annals of Photogrammetry, Remote Sensing and Spatial Information Sciences, IV-2/W4, 355362. https://doi.org/10.5194/isprs-annals-IV-2-W4-355-2017

Nocerino E, Menna F, Remondino F, Toschi I, Rodríguez-Gonzálvez P (2017). Investigation of indoor and outdoor performance of two portable mobile mapping systems. In F. Remondino \& M. R. Shortis (Eds.), Videometrics, Range Imaging, and Applications XIV, edited, SPIE 2017 (p. 103320I). https://doi.org/10.1117/12. 2270761

Ottoni F, Rodriguez A, Spanò AT (2017). La documentazione dei complessi monumentali. In ANANKE Speciale GEORES 2017 (pp. 13-14)

Puente I, González-Jorge H, Martínez-Sánchez J, Arias P (2013) Review of mobile mapping and surveying technologies. Measurement 46(7):2127-2145. https://doi.org/10.1016/j.measurement.2013.03. 006

Remondino F, Spera MG, Nocerino E, Menna F, Nex F (2014) State of the art in high density image matching. Photogramm Rec 29(146): 144-166. https://doi.org/10.1111/phor.12063

Riisgaard S (2005). SLAM for dummies: a tutorial approach to simultaneous localization and mapping. Citeulike.org. https://doi.org/10. $1017 / \mathrm{S} 0025315400002526$

Rodríguez-Gonzálvez P, Jiménez Fernández-Palacios B, Muñoz-Nieto Á, Arias-Sanchez P, Gonzalez-Aguilera D (2017) Mobile LiDAR system: new possibilities for the documentation and dissemination of large cultural heritage sites. Remote Sens 9(3):189. https://doi.org/ $10.3390 / \mathrm{rs} 9030189$

Ryding J, Williams E, Smith M, Eichhorn M (2015) Assessing handheld mobile laser scanners for forest surveys. Remote Sens 7(1):10951111. https://doi.org/10.3390/rs70101095

Sammartano G (2017). Optimization of 3D multi-sensor models for damage assessment in emergency context: first tests on rapid mapping in the 2016 Italian earthquake. In F. Remondino, A. Georgopoulos, D. Gonzalez-Aguilera, \& P. Agrafiotis (Eds.), Latest Developments in Reality-Based 3D Surveying and Modeling. MDPI

Sammartano G (2018) Suitability of 3D dense models for rapid mapping strategies on cultural heritage documentation and conservation. 
Validation of metric and non-metric information extraction from integrated solutions. $\mathrm{PhD}$ thesis, Politecnico di Torino

Sirmacek B, Shen Y, Lindenbergh R, Zlatanova S, Diakite A (2016). Comparison of ZEB1 and LEICA C10 indoor laser scanning point clouds. ISPRS Annals of Photogrammetry, Remote Sensing and Spatial Information Sciences, III-1, 143-149. https://doi.org/10. 5194/isprsannals-III-1-143-2016

Thomson C, Apostolopoulos G, Backes D, Boehm J (2013). Mobile laser scanning for indoor modelling. ISPRS Annals of Photogrammetry, Remote Sensing and Spatial Information Sciences, II-5/W2, 289293. https://doi.org/10.5194/isprsannals-II-5-W2-289-2013

Toschi I, Ramos MM, Nocerino E, Menna F, Remondino F, Moe K, ... Fassi F (2017). Oblique photogrammetry supporting 3D urban reconstruction of complex scenarios. ISPRS - International Archives of the Photogrammetry, Remote Sensing and Spatial Information Sciences, XLII-1/W1, 519-526. https://doi.org/10.5194/isprsarchives-XLII-1-W1-519-2017

Toschi I, Rodríguez-Gonzálvez P, Remondino F, Minto S, Orlandini S, Fuller A (2015). Accuracy evaluation of a mobile mapping system with advanced statistical methods. ISPRS-International Archives of the Photogrammetry, Remote Sensing and Spatial Information Sciences, XL-5/W4, 245-253. https://doi.org/10.5194/ isprsarchives-XL-5-W4-245-2015

Tucci G, Bonora V, Conti A, Fiorini L (2017). Digital workflow for the acquisition and elaboration of 3D data in a monumental complex: the fortress of Saint John the Baptist in Florence. ISPRSInternational Archives of the Photogrammetry, Remote Sensing and Spatial Information Sciences, XLII-2/W5, 679-686. https:// doi.org/10.5194/isprs-archives-XLII-2-W5-679-2017

Vanneschi C, Eyre M, Francioni M, Coggan J (2017) The use of remote sensing techniques for monitoring and characterization of slope instability. Procedia Engineering 191:150-157. https://doi.org/10. 1016/j.proeng.2017.05.166

Zlot R, Bosse M, Greenop K, Jarzab Z, Juckes E, Roberts J (2014) Efficiently capturing large, complex cultural heritage sites with a handheld mobile 3D laser mapping system. J Cult Herit 15(6): 670-678. https://doi.org/10.1016/j.culher.2013.11.009 